\title{
On the Very Weak 0-1 Law for Random Graphs with Orders
}

\author{
Saharon Shelah \\ The Hebrew University, Math Institute \\ Rutgers University, Math Department
}

Abstract: Let us draw a graph $R$ on $\{0,1, \ldots, n-1\}$ by having an edge $\{i, j\}$ with probability $p_{|i-j|}$, where $\sum_{i} p_{i}<\infty$, and let $M_{n}=(n,<, R)$. For a first order sentence $\psi$ let $a_{\psi}^{n}$ be the probability of $M_{n} \models \psi$. We know that the sequence $a_{\psi}^{1}, a_{\psi}^{2}, \ldots, a_{\psi}^{n}, \ldots$ does not necessarily converge. But here we find a weaker substitute which we call the very weak $0-1$ law. We prove that $\lim _{n \rightarrow \infty}\left(a_{\psi}^{n}-a_{\psi}^{n+1}\right)=0$. For this we need a theorem on the (first order) theory of distorted sum of models.

Research partially supported by the Binational Science Foundation and partially sponsored by the Edmund Landau Center for research in Mathematical Analysis, supported by the Minerva Foundation (Germany). Done fall 91, Publication 463. 


\section{$\S 0$ Introduction}

The kind of random models $M_{n}=(n,<, R)$ from the abstract are from Luczak Shelah [LuSh 435] where among other things, it is proved that the probability $a_{\psi}^{n}=: \operatorname{Prob}\left(M_{n} \models \psi\right)$ of $M_{n} \models \psi$ does not necessarily converge (but if $\sum_{i} i p_{i}<\infty$ then it converges, and the value of $\sum p_{i}$ is not enough, in general, to determine convergence). The theorem in the abstract appears in $\S 1$ and is proved in $\S 3$, it says that the sequence of probabilities still behaves (somewhat) nicely.

The first results (in various probabilistic distributions) on the asymptomatic behavior of $a_{\psi}^{n}$ (see Glebski et al [GKLT], Fagin [F] and survey [Sp]) say that it converges to 0 or 1 hence the name zero one law. In other cases weaker results were gotten: $a_{\psi}^{n}$ converges (to some real). We suggest an even weaker version: $\left|a_{\psi}^{n+1}-a_{\psi}^{n}\right|$ converges to zero. We also define $h$-very weak zero one law (see Definition 1.2(2)), but concentrate on the one above. Note that many examples to nonconvergence are done by finding $\psi$ such that e.g. if $\log (\mathrm{n})=1 \bmod 10$ then $a_{\psi}^{n} \sim 1$ and if $\log (\mathrm{n})=5 \bmod 10$ then $a_{\psi}^{n} \sim 5$ (or even using functions with $h(n) \rightarrow \infty, h(n)<<\log (\mathrm{n}))$. As the most known results were called zero one law we prefer the name "very weak 0-1 law" on weak convergence. A (first order) sentence whose probability $a_{\psi}^{n}$ (defined above, for the distribution defined above) may not converge (by [LuSh 435]) is $\psi_{0}=:(\exists x) \forall y z(y<x \leq z \rightarrow \neg y R z)$. But we can find a sequence $m_{0}<m_{1}<\ldots$ such that the probability that $\Phi=: \bigvee_{i}(\exists y z)\left(y \leq m_{i} \& m_{i+1} \leq z \& y R z\right)$ is very small. How do we prove $0=\lim \left(a_{\psi}^{n}-a_{\psi}^{n+1}\right)$ ? By changing the rule of making the random choice we get $M_{n}^{\prime}$ nice enough, ensuring $\Phi$ holds, while the probability changes little (see $\S 3)$. Now $M_{n}^{\prime}$ is almost the sum of $M_{n}^{\prime} \uparrow\left(m_{i}, m_{i+1}\right)$, precisely $M_{n}^{\prime}$ is determined by $M_{n}^{\prime} \uparrow\left[m_{i}, m_{i+2}\right)$, for $i=0,1,2, \ldots$, so we call it a distorted sum. Now a model theoretic lemma from $\S 2$ on the $n$-theory of a distorted sum of models enables us to prove the main theorem in $\S 3$ (on the model theoretic background see $\S 2$ ). Later in $\S 4, \S 5$ we deal with some refinements not needed for the main theorem (1.4).

In $§ 1$ we also get the very weak 0-1 law for a random partial order suggested by Luczak. In a subsequent paper [Sh 548] we prove the very weak zero law for some other very natural cases: e.g. for a random 2-place function and for $(n,<, R)$ with $<$ the natural order, $R$ a random graph (=symmetric irreflexive relation) with edge probability $p$. In another one, [Sh 467] we deal with zero one law for the random model from the abstract with $p_{i}=\frac{1}{i^{a}}$ (mainly: no order, $a \in(0,1)$ irrational). See also [Sh 550], [Sh 551], [Sh 581]. Spencer is continuing [Sh 548] 
looking at the exact $h$ for which $h$-very weak zero one law holds (see Definition 1.2(2) here). I thank Shmuel Lifsches for many corrections.

\section{Notation}

$\mathbb{N}$ is the set of natural numbers.

We identify $n \in \mathbb{N}$ with the set $\{0, \ldots, n-1\}$.

$\mathbb{Z}$ is the set of integers.

$\mathbb{R}$ is the set of reals, $\mathbb{R}^{+}$is the set of reals which are positive (i.e. $>0$ ).

$i, j, k, \ell, m, n, r, s, t$ are natural numbers.

$\varepsilon, \zeta$ are positive reals (or functions with values in $\mathbb{R}^{+}$).

$f, g, h$ are functions.

$\tau$ denotes a vocabulary (for simplicity- set of predicates), $\mathcal{L}_{\tau}^{\text {fo }}$ is the set of first order formulas in the vocabulary $\tau$. (Generally, if $\mathcal{L}$ is a logic $\mathcal{L}_{\tau}$ the set of sentences (or formulas) in $\mathcal{L}$ in the vocabulary $\tau$ and $\mathcal{L}^{\text {fo }}$ is the first order $\operatorname{logic})$.

For a first order sentence (or formula) $\varphi$ let $d_{\varphi}=d[\varphi]$ be its quantifier depth. $M, N$ denote models, but we do not distinguish strictly between a model and its universe $=$ set of elements.

$\tau(M)$ is the vocabulary of $M$, for $R \in \tau(M), n(R)$ is the number of places (=arity of $R$ ), $R^{M}$ the interpretation of $R$ in $M$.

A basic formula is one of the form $\pm R\left(x_{i_{0}}, \ldots, x_{i_{n(R)-1}}\right)$ (i.e. $R\left(x_{i_{0}}, \ldots, x_{i_{n(R)-1}}\right)$ or $\neg R\left(x_{i_{0}}, \ldots, x_{i_{n(R)-1}}\right)$

$\bar{a}$ denotes a sequence of elements of a model. $\ell g(\bar{a})$ is the length of $\bar{a}$.

If $<$ belongs to the vocabulary $\tau$, then in $\tau$-models $<^{M}$ is a linear order, if not said otherwise. If $M_{i}$ are $\tau$-models for $i<n, M=\sum_{i<n} M_{i}$ is (assuming for simplicity the universes are pairwise disjoint) the models defined by: universe $\bigcup_{i<n} M_{i}, R^{M}=\bigcup_{i<n} R^{M_{i}}$ for $R \in \tau$ except that if $<\in \tau$ then: $x<{ }^{M} y \Leftrightarrow$ $\bigvee_{i<j<n}\left[x \in M_{i} \& y \in M_{j}\right] \vee \bigvee_{i<n} x<{ }^{M_{i}} y$ (similarly with any linear order $I$ instead of $n$ ). We write $M_{0}+M_{1}$ instead of $\sum_{i<2} M_{i}$.

$\psi^{\text {if }(\theta)}$ is $\psi$ if $\theta$ is true, $\neg \psi$ if $\theta$ is false.

We identify true, false with yes, no.

Note: $t$ is a natural number, $\mathbf{t}$ kind of depth $n$ theory, $\mathfrak{t}$ is a truth value, $\boldsymbol{\Delta}$ is the symmetric difference, $\Delta$ denote a set of formulas.

\section{$\S 1$ The Very Weak Zero One Law.}


1.1 Definition: 1) A 0-1 law context is a sequence $\bar{K}=\left\langle K_{n}, \mu_{n}: n<\omega\right\rangle$ such that:

(a) for some vocabulary $\tau=\tau_{\bar{K}}$, for every $n, K_{n}$ is a family of $\tau$-models, closed under isomorphism with the family of isomorphism types being a set.

(b) for each $n, \mu_{n}$ is a probability measure on the set of isomorphism types (of models from $K_{n}$ ).

2) $\bar{K}$ is finitary (countable) if for each $n$ the set $\left\{M / \cong: M \in K_{n}\right\}$ is finite (countable).

3) For a sentence $\psi$ (not necessarily f.o.) $\operatorname{Prob}_{\mu_{n}}\left(M_{n} \models \psi\right)$ or $\operatorname{Prob}_{K_{n}}\left(M_{n} \models \psi\right.$ ) or $\operatorname{Prob}_{\mu_{n}}\left(M_{n} \models \psi \mid M_{n} \in K_{n}\right)$ means $\mu_{n}\left\{M / \cong: M \in K_{n}, M \models \psi\right\}$.

4) Instead of clause (a) of (1), we may use $K_{n}$ a set of $\tau$-models, $\mu_{n}$ a probability measure on $K_{n}$; particularly we introduce a random choice of $M_{n}$; the translation between the two contexts should be clear.

1.1A Discussion: A 0-1 law context is not necessarily a context which satisfies a 0-1 law. It is a context in which we can formulate a 0-1 law, and also weaker variants.

1.2 Definition: 0 ) A $0-1$ context $\bar{K}$ satisfies the $0-1$ law for a logic $\mathcal{L}$ if for every sentence $\varphi \in \mathcal{L}_{\tau}$ (with $\tau=\tau_{\bar{K}}$ of course) we have: $a_{\varphi}^{n} \stackrel{\text { def }}{=} \operatorname{Prob}_{\mu_{n}}\left(M_{n} \models \varphi\right)$ converges to zero or converges to 1 when $n \rightarrow \infty$.

1) $\bar{K}$ satisfies the very weak $0-1$ law for the logic $\mathcal{L}$ if for every sentence $\varphi \in \mathcal{L}_{\tau}$ we have: $a_{n} \stackrel{\text { def }}{=} \operatorname{Prob}_{\mu_{n}}\left(M_{n+1} \models \varphi \mid M_{n+1} \in K_{n+1}\right)-\operatorname{Prob}_{\mu_{n}}\left(M_{n} \models \varphi \mid M_{n} \in K_{n}\right)$ converges to zero as $n \rightarrow \infty$.

2) $\bar{K}$ satisfies the $h$-very $0-1$ one law for the logic $\mathcal{L}$ if for every sentence

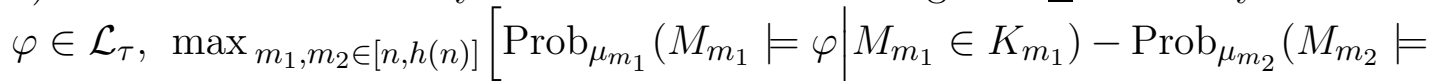
$\left.\left.\varphi \mid M_{m_{2}} \in K_{m_{2}}\right)\right]$ converge to zero as $n \rightarrow \infty$. (We shall concentrate on part 1 ). 3) $\bar{K}$ satisfies the convergence law for $\mathcal{L}$ if for every $\varphi \in \mathcal{L}_{\tau}$ we have: $\left\langle\operatorname{Prob}_{\mu_{n}}\left(M_{n} \models\right.\right.$ $\left.\left.\varphi \mid M_{n} \in K_{n}\right): n<\omega\right\rangle$ converges (to some real $\in[0,1]_{\mathbb{R}}$ ). (So if it always converges to 0 or to 1 we say that $\bar{K}$ satisfies the $0-1$ law for $\mathcal{L}$ ).

4) If $\mathcal{L}$ is the first order logic we may omit it.

The following $\bar{K}$ is from Luczak Shelah [LuSh 435].

1.3 Definition: Let $\bar{p}=\left\langle p_{i}: i \in \mathbb{N}\right\rangle, p_{i}$ a real number $0 \leq p_{i} \leq 1, p_{0}=0$. We define $\bar{K}_{\bar{p}}^{\mathrm{og}}$ as follows:

the models from $K_{n}$ are of the form $M=(n,<, R)$, so we are using the variant 
from $^{\dagger}$ Definition 1.1(4) where $n=\{0, \ldots, n-1\},<$ the usual order, $R$ a graph (i.e. a symmetric relation on $n$ which is irreflexive i.e. $\neg x R x)$ and: $\operatorname{Prob}_{\mu_{n}}\left(M_{n} \cong\right.$ $\left.M \mid M_{n} \in K_{n}\right)$ is $\prod\left\{p_{j-i}: i<j<n\right.$ and $\left.i R j\right\} \times \prod\left\{1-p_{j-i}: i<j<n\right.$ and $\neg i R j\}$, i.e. for each $i<j<n$ we decide whether $i R j$ by flipping a coin with probability $p_{j-i}$ for yes, independently for the different pairs.

1.4 Theorem: $\bar{K}^{\mathrm{og}}$ satisfies the very weak $0-1$ law if $\sum_{i} p_{i}<\infty$.

This is our main result. We shall prove it later in $§ 3$. Luczak [Lu] suggested another context:

1.5 Definition: $\bar{K}_{p_{n}}^{\text {opo }}$ is the following 0-1 law context $\left(p_{n}\right.$ is a function of $n$, $0 \leq p_{n} \leq 1$; so more precisely we are defining $\left.\left\langle K_{p_{n}}^{\text {opo }}: n<\omega\right\rangle\right)$. The models are of the form, $\left(n,<,<^{*}\right), n=\{0,1, \ldots, n-1\}$, <-the usual order, $<^{*}$-the following partial order: we draw a random graph on $n$ (edge relation $\mathbb{R}$ ) with edge probability $p_{n}, x<^{*} y$ iff there are $k \in N$ and $x=x_{0}<x_{1}<\ldots<x_{k}=y<n$ such that $x_{\ell} R x_{\ell+1}$. The probability is derived from the probability distribution for $R$ (which does not appear in the model.)

1.6 Theorem Assume $p_{n}=\frac{1}{(n+1)^{a}}$, where $0<a<1$ (like [ShSp 304]). Then $\bar{K}_{p_{n}}^{\text {opo }}$ satisfies the very weak zero one law.

Proof: Similar to the previous theorem as easily

(*) for $\varepsilon>0$ we have: $\operatorname{Prob}\left(M_{n} \models(\exists x<y)\left[\neg x<<^{*} y \& x+n^{1-\varepsilon} \leq y\right)\right.$ is very small.

\section{$\S 2$ Model Theory: Distorted sum of Models}

The main lemma (2.14) generalizes the addition theory and deals with models with distances (both from model theory). Concerning the first, see Feferman Vaught [FV]. The method has its origin in Mostowski [Mo], who dealt with reduced products. The first work on other products is Beth [B] who dealt with the ordinal sum of finitely many ordered systems. For a presentation and history see Feferman Vaught [FV], pp 57-59 and Gurevich [Gu] (particularly the $\mathrm{th}^{n}$ 's). Concerning models with distance see Gaifman [Gf], a forerunner of which was Marcus [M1] who deals with the case of $M=\left(M, F, P_{i}\right)_{i<n}, F$ a unary function, $P_{i}$ unary predicates and the distance is as in the graph which the function $F$

$\dagger$ but no two models are isomorphic so the difference is even more trivial 
defines (i.e. $x, y$ connected by an edge if: $x=F(y)$ or $y=F(x)$; where for a graph $G$ the distance is $d_{G}(x, y)=\min \left\{k\right.$ : we can find $x_{0}, \ldots, x_{k}$ such that: $x=x_{0}, y=x_{k}$, and $x_{\ell}, x_{\ell+1}$ are connected $\}$ ). We may look at our subject here as dealing with sums with only local "disturbances", "semi sums"; "distorted sums". The connections are explained in $\S 4, \S 5$.

In 2.16 we draw the conclusion for linear order which we shall use in $\S 3$ to prove theorem 1.4, in fact proving the main theorem 1.4 from $\S 2$ we use almost only 2.16. Elsewhere we shall return to improving the numerical bound involved in the proof see [Sh, F-120]

Note: $\mathfrak{B}$ is a (two sorted) model.

2.1 Definition: 1) We call $\sigma$ a vocabulary of systems if $\sigma=\left\langle\tau_{1}, \tau_{2}\right\rangle, \tau_{1}, \tau_{2}$ are sets of predicates (usually finite but not needed).

2) We call $\mathfrak{B}$ a $\sigma$-system if:

(A) $\mathfrak{B}=(M, I, h, d)\left(=\left(M^{\mathfrak{B}}, I^{\mathfrak{B}}, h^{\mathfrak{B}}, d^{\mathfrak{B}}\right)\right)$.

(B) $M$ is a $\tau_{1}$-model and $I$ is a $\tau_{2}$-model, but we use $M, I$ also for their universes.

(C) $h$ is a function from $M$ onto $I$,

(D) $d$ is a distance function on $I$, i.e.

$(\alpha) d$ is a symmetric two place function from $I$ to $\mathbb{N} \cup\{\infty\}=\{0,1,2,3, \ldots, \infty\}$,

( $\beta) d(x, x)=0$

$(\gamma) d(x, z) \leq d(x, y)+d(y, z)$.

(E) $M, I$ are disjoint.

3) Let $\sigma(\mathfrak{B})=\sigma$ for $\mathfrak{B}$ a $\sigma$-system and $\tau_{\ell}(\mathfrak{B})=\tau_{\ell}$ if $\sigma=\left\langle\tau_{1}, \tau_{2}\right\rangle$.

2.1A Discussion: The demands " $M, I$ are disjoint and $h$ is onto $I$ " are not essential, this is just for convenience of presentation. If $h$ is not onto $I$, we should allow relations on $M \cup I$, but then if $M, I$ are not disjoint then for each predicate, for each of its places we should assign: does there appear a member of $M$ or a member of $I$; also we should have two kinds of variables not allowing equality between variables of the different kinds. So in application we may use those alternative presentations.

2.2 Conventions: (1) We may allow function symbols (and individual constants) but then treat them as relations.

(2) We stipulate $h(x)=x$ for $x \in I$ (remember 2.1(2)(E)).

(3) $\bar{a} \subseteq \mathfrak{B}$ means $\operatorname{Rang}(\bar{a}) \subseteq M^{\mathfrak{B}} \cup I^{\mathfrak{B}}$. 
(4) A model $M$ will be identified with the system $\mathfrak{B}=\mathfrak{B}^{\operatorname{sim}}[M]: M^{\mathfrak{B}}=M$, $I^{\mathfrak{B}}=M, h^{\mathfrak{B}}=\mathrm{id}_{M}$,

$$
d(x, y)= \begin{cases}0 & x=y \\ \infty & x \neq y\end{cases}
$$

(you may of course take two disjoint copies of $M$ as $M^{\mathfrak{B}}$ and $I^{\mathfrak{B}}$ ).

(5) From a model $M$ we can derive another system $\mathfrak{B}^{\operatorname{dis}}[M]: M^{\mathfrak{B}}=M, I^{\mathfrak{B}}=M$, $h^{\mathfrak{B}}=\operatorname{id}_{M}$, and $d(x, y) \stackrel{\text { def }}{=} \operatorname{Min}\left\{n:\right.$ there are $z_{0}, \ldots, z_{n} \in M, x=z_{0}, y=z_{n}$ and for $\ell<n$ for some $R \in \tau(M)$, and sequence $\bar{a} \in R^{M}$ we have $\left.\left\{z_{\ell}, z_{\ell+1}\right\} \subseteq \operatorname{Rang} \bar{a}\right\}$ (remember $\tau(M)$ is the vocabulary of $M$; this is the definition of distance in Gaifman [Gf]).

2.2A Remark: 1) So the difference between $\mathfrak{B}^{\operatorname{sim}}[M]$ and $\mathfrak{B}^{\text {dis }}[M]$ is only in the choice of the distance function $d$.

2) Below in $\mathcal{L}_{\sigma_{0}}$ the formula $d(x, y)=0$ appears which normally means $x=y$. if not we could have in Def. $2.3(3)$, (4) replaced " $k \leq r$ ", " $s \leq n$ " by " $k<r$ ", " $s<n$ " respectively.

2.3 Definition: 1$)$ For a system $\mathfrak{B}=(M, I, h, d)$ :

for $x \in I$,

$$
N_{r}(x) \stackrel{\text { def }}{=}\{y \in I: d(y, x) \leq r\}
$$

for $x \in M \cup I$,

$$
N_{r}^{+}(x) \stackrel{\text { def }}{=}\{y \in M \cup I: d(h(y), h(x)) \leq r\} .
$$

2) $\mathcal{L}_{\sigma}$ is the set of first order formulas for $\sigma$; we have variables on $M$, variables on $I$, the predicates of $\tau_{1}, \tau_{2}$; and the additional atomic formulas $h(x)=y$; and " $h(y) \in N_{r}(h(x))$ " for each $r$ (see part (4) below).

3) $\mathfrak{B}^{-}=\left(M^{\mathfrak{B}}, I^{\mathfrak{B}}, h\right), \mathfrak{B}_{r}=\left(M^{\mathfrak{B}}, I^{\mathfrak{B}}, h^{\mathfrak{B}}, " d(x, y) \leq k^{\prime \prime}\right)_{k \leq r}$ so $\mathfrak{B}_{0}=\mathfrak{B}^{-}$and we let $\sigma_{r}=\sigma_{r}(\mathfrak{B})=\sigma\left(\mathfrak{B}_{r}\right)$ (so, $\mathfrak{B}_{r}$ is a two sorted model but not a system).

4) So $\mathcal{L}_{\sigma_{n}}$ is defined as in part (2) but " $h(y) \in N_{s}(h(x))$ " appear only for $s \leq n$.

We usually apply our theorems in the following case:

2.4 Definition: A system $\mathfrak{B}$ is simple if

(a) $d(x, y) \leq 1$ (where $x, y$ vary on $I$ ) is equivalent to a quantifier free formula in $\mathcal{L}_{\sigma_{0}(\mathfrak{B})}$.

(b) for $x, y \in I$ we have: $d(x, y) \leq r$ iff there are $x_{0}, \ldots, x_{r} \in I$ such that $x=x_{0}$, $y=x_{r}$, and $d\left(x_{\ell}, x_{\ell+1}\right) \leq 1$ for $\ell<r$ (i.e., like $\left.2.2(5)\right)$. 
2.4A Remark: If $\mathfrak{B}$ is simple, note that every formula in $\mathcal{L}_{\sigma(\mathfrak{B})}$ is equivalent to some formula in $\mathcal{L}_{\sigma_{0}}$, if we know just that clause (b) of Definition 2.4 is satisfied then every formula in $\mathcal{L}_{\sigma(\mathfrak{B})}$ is equivalent to some formula in $\mathcal{L}_{\sigma_{1}}$.

2.5 Convention: 1) We define $f$ : we let $f_{n}(r)=r+3^{n}$ for $r, n \in \mathbb{N}$ or more generally, $f$ a two place function (written $f_{n}(r)$ ) from $\mathbb{N}$ to $\mathbb{N}$ satisfying: $f_{n}$ non decreasing in $n$ and in $r, r<f_{n}(r) \in \mathbb{N}$ and $f_{n}^{(3)}(r) \leq f_{n+1}(r)$ where $f_{n}^{(0)}(r)=r$, $f_{n}^{(\ell+1)}(r)=f_{n}\left(f_{n}^{(\ell)}(r)\right)$ and $f_{n}^{(2)}(r) \geq f_{n}(r)+f_{n}(0)$.

2) We call $f$ nice if in addition $f_{n}^{(4)}(r) \leq f_{n+1}(r)$.

3) For $g$ a function from $\mathbb{N}$ to $\mathbb{N}$, let $g\left(\left\langle r_{\ell}: \ell<m\right\rangle\right)=\left\langle g\left(r_{\ell}\right): \ell<m\right\rangle$.

2.6 Definition: 1) For a system $\mathfrak{B}=(M, I, h, d)$ and $m, n \in \mathbb{N}$, and $\bar{a}=$ $\left\langle a_{0}, \ldots, a_{m-1}\right\rangle \subseteq \mathfrak{B}$ we define $\operatorname{th}_{r}^{n}(\bar{a}, \mathfrak{B})$, here $r$ stands for a distance. We define it by induction ${ }^{\dagger}$ on $n$ :

$$
\begin{aligned}
& \operatorname{th}_{r}^{0}(\bar{a}, \mathfrak{B}) \text { is }\left\{\varphi\left(x_{0}, \ldots, x_{m-1}\right):(M, I, h) \models \varphi\left[a_{0}, \ldots, a_{m-1}\right]\right. \text {, } \\
& \left.\varphi \text { a basic formula in } \mathcal{L}_{\sigma\left(\boldsymbol{B}_{r}\right)}\right\} \\
& \operatorname{th}_{r}^{n+1}(\bar{a}, \mathfrak{B})=\left\{\operatorname{th}_{r}^{n}(\bar{a} \widehat{ }\langle c\rangle, \mathfrak{B}): c \in M \cup I\right\} .
\end{aligned}
$$

2) If $\sigma$ is a vocabulary of systems, $n, m \in \mathbb{N}$, then $\operatorname{TH}_{r}^{n}(m, \sigma)$ is the set of formally possible $\operatorname{th}_{r}^{n}(\bar{a}, \mathfrak{B})$ for $\mathfrak{B}$ a $\sigma$-system, $\bar{a} \subseteq \mathfrak{B}$ and $\lg (\bar{a})=m$; this is defined naturally. Pedantically, we define it by induction on $n$; for $n=0$ it is the family of sets $\mathbf{t}$ of basic formulas $\varphi\left(x_{0}, \ldots, x_{m-1}\right)$ of $\mathcal{L}_{\sigma_{r}}$ such that for each atomic $\varphi\left(x_{0}, \ldots, x_{m-1}\right)$ exactly one of $\varphi\left(x_{0}, \ldots, x_{m-1}\right), \neg \varphi\left(x_{0}, \ldots, x_{m-1}\right)$ belongs to $\mathbf{t}$. $\mathrm{TH}_{r}^{n+1}(m, \sigma)$ is the family of subsets of $\operatorname{TH}_{r}^{n}(m+1, \sigma)$.

3) If $\tau$ is a vocabulary of models (see 2.2(4)), $n, m \in \mathbb{N} \underline{\text { then }} \mathrm{TH}^{n}(m, \tau)$ is the set of formally possible $\operatorname{th}_{0}^{n}(\bar{a}, \mathfrak{B}), \mathfrak{B}$ a $\tau$-model, i.e. $\mathfrak{B}=\mathfrak{B}^{\operatorname{sim}}[M]$ for some $\tau$-model $M$ (note: the value of $r$ is immaterial as the distance function is trivial).

4) If $r=0$ we may omit it, so for a model $M$, using $I=M, h$ the identity we get the usual $t h^{n}(\bar{a}, M)$ (but we do not assume knowledge about it).

5) If $\bar{a}$ is empty sequence we may omit it.

2.7 Claim: 1) For $\mathfrak{B}, n, m, \bar{a}$ as above, $\varphi=\varphi\left(x_{0}, \ldots, x_{m-1}\right)$ a (first order) formula in $\mathcal{L}_{\sigma\left(\mathfrak{B}_{r}\right)}$ of quantifier depth $n$, we have:

from $\operatorname{th}_{r}^{n}(\bar{a}, \mathfrak{B})$ we can compute the truth value of " $\mathfrak{B}_{r} \models \varphi[\bar{a}]$ ".

Here and in later instance we mean:

for any $\mathbf{t} \in \mathrm{TH}_{r}^{n}(m, \sigma)$ we can compute a truth value $\mathfrak{t}$ such that: if $\mathbf{t}=\operatorname{th}_{r}^{n}(\bar{a}, \mathfrak{B})$

$\dagger$ In the following definition basic is atomic or a negation of atomic. 
then $\mathfrak{t}$ is the truth value of " $\mathfrak{B}_{r} \models \varphi[\bar{a}]$ ". Also in the proof we behave similarly. 2) For any $\sigma$ and $r, n, m \in \mathbb{N}$, if $\mathbf{t} \in \operatorname{TH}_{r}^{n}(m, \sigma)$ then for some formula $\varphi\left(x_{0}, \ldots, x_{m-1}\right) \in \mathcal{L}_{\sigma_{r}}$ of quantifier depth $n$, for any $\sigma$-system $\mathfrak{B}$ and $\bar{a}=$ $\left\langle a_{0}, \ldots, a_{m-1}\right\rangle \subseteq \mathfrak{B}$ we have: $\mathbf{t}=\operatorname{th}_{r}^{n}(\bar{a}, \mathfrak{B})$ iff $\mathfrak{B} \models \varphi[\bar{a}]$.

3) The functions $\langle\sigma, n, m, r\rangle \mapsto \mathrm{TH}_{r}^{n}(m, \sigma)$ and $\langle\tau, n, m\rangle \mapsto \mathrm{TH}^{n}(m, \tau)$ are computable.

4) From $\operatorname{th}_{r}^{n}(\bar{a}, \mathfrak{B})$ we can $\operatorname{compute} \operatorname{th}_{s}^{m}(\bar{a}, \mathfrak{B})$ if $r \geq s$ and $n \geq m$. Also if $\operatorname{Rang}(\bar{b}) \subseteq \operatorname{Rang}(\bar{a})$ then from $\operatorname{th}_{r}^{n}(\bar{a}, \mathfrak{B})$ and $\left\{(\ell, m): b_{\ell}=a_{m}\right\}$ we can compute $\operatorname{th}_{r}^{n}(\bar{b}, \mathfrak{B})$. (See part (1).)

$5)$ If $\mathfrak{B}$ is simple then from $\operatorname{th}_{1}^{n+r}(\bar{a}, \mathfrak{B})$ we can compute $\operatorname{th}_{2^{r}}^{n}(\bar{a}, \mathfrak{B})$.

6) If $n_{1}, n_{2} \geq 2^{d} \underline{\text { then }} \operatorname{th}^{d}\left(\langle\rangle,\left(n_{1},<\right)\right)=\operatorname{th}^{d}\left(\langle\rangle,\left(n_{2},<\right)\right)$. Also if $\operatorname{th}^{d}\left(\langle\rangle, M_{i}^{\ell}\right)=\mathbf{t}$ for $\ell=1,2$ and for $i<\max \left\{n_{1}, n_{2}\right\}, \tau$ a vocabulary, $M_{i} \in K_{\tau}$ then $\operatorname{th}^{d}\left(\langle\rangle, \sum_{i<n_{1}} M_{i}^{1}\right)=\operatorname{th}^{d}\left(\langle\rangle, \sum_{i<n_{2}} M_{i}^{2}\right)$. If $M_{i}^{\ell} \in K_{\tau}$ for $\ell=1,2, i<k$ and $\operatorname{th}^{d}\left(M_{i}^{1}\right)=\operatorname{th}^{d}\left(M_{i}^{2}\right)$ for $i<k$ then $\operatorname{th}^{d}\left(\langle\rangle, \sum_{i<k} M_{i}^{1}\right)=\operatorname{th}^{d}\left(\langle\rangle, \sum_{i<k} M_{i}^{2}\right)$.

7) For a given vocabulary $\tau$, and $d \in \mathbb{N}$ there is an operation $\oplus$ on $\operatorname{TH}^{d}(0, \tau)$ such that $\operatorname{th}^{d}\left(\langle\rangle, \sum_{i<k} M_{i}\right)=\oplus\left\langle\operatorname{th}^{d}\left(\langle\rangle, M_{i}\right): i<k\right\rangle$, this operation is associative (but in general not commutative).

Proof: 1) We prove this by induction on the formula. (It goes without saying that the reasoning below does not depend on $\mathfrak{B}$.)

$\varphi$ atomic: Thus $n=d(\varphi)=0$, and by the Definition of $\operatorname{th}_{r}^{n}(\bar{a}, \mathfrak{B})$ the statement is trivial.

$\varphi=\neg \psi$ : Easy by the induction hypothesis.

$\varphi=\varphi_{1} \wedge \varphi_{2}\left(\right.$ or $\varphi_{1} \vee \varphi_{2}$, or $\left.\varphi_{1} \rightarrow \varphi_{2}\right)$ : Easy by the induction hypothesis.

$\varphi=(\exists x) \varphi_{1}$ : Without loss of generality $\varphi=\left(\exists x_{m}\right) \varphi_{1}\left(x_{0}, \ldots, x_{m-1}, x_{m}\right)$. So $d\left(\varphi_{1}\right)=n-1$, and by the induction hypothesis for $a_{0}, \ldots, a_{m} \in \mathfrak{B}$ we have: the truth value of $\mathfrak{B} \models \varphi_{1}\left[a_{0}, \ldots, a_{m-1}, a_{m}\right]$ is computable from $\operatorname{th}_{r}^{n-1}\left(\left\langle a_{0}, \ldots, a_{m}\right\rangle, \mathfrak{B}\right)$. Say it holds iff $\operatorname{th}_{r}^{n-1}\left(\left\langle a_{0}, \ldots, a_{m}\right\rangle, \mathfrak{B}\right) \in \mathbf{T}_{\varphi_{1}}\left(\mathbf{T}_{\varphi_{1}}\right.$ a subset of $\operatorname{TH}_{r}^{n-1}(m+$ $1, \sigma(\mathfrak{B})))$.

Now $\mathfrak{B} \models \varphi\left[a_{0}, \ldots, a_{m-1}\right]$ iff for some $a_{m}, \mathfrak{B} \models \varphi_{1}\left[a_{0}, \ldots, a_{m-1}, a_{m}\right]$, iff $\operatorname{th}_{r}^{n-1}\left(\left\langle a_{0}, \ldots, a_{m-1}, a_{m}\right\rangle, \mathfrak{B}\right) \in \mathbf{T}_{\varphi_{1}}\left(\mathbf{T}_{\varphi_{1}}\right.$ the subset of $\mathrm{TH}_{r}^{n-1}(m+1, \sigma(\mathfrak{B}))$ from

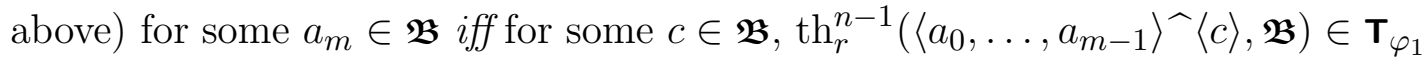
iff $\operatorname{th}_{r}^{n}\left(\left\langle a_{0}, \ldots, a_{m-1}\right\rangle, \mathfrak{B}\right)$ is not disjoint to $\mathbf{T}_{\varphi_{1}}$ ( the first "iff" by the definition of satisfaction, the second "iff" by the choice of $\mathbf{T}_{\varphi_{1}}$, the third "iff" is trivial; the last "iff" by the induction step in the definition of $\operatorname{th}_{r}^{n}$ ). So we have completed 
the induction.

2) We define $\varphi=\varphi_{\mathbf{t}}$ for $\mathbf{t} \in \mathrm{TH}_{r}^{n}(m, \sigma)$ as required by induction on $n$; check the inductive definition of $\operatorname{th}_{r}^{n}$.

3) Read the definition. (2.6(2))

4) By induction on $n$, for $n=0$ as $\operatorname{th}_{r}^{n}$ "speaks" on more basic formulas. For $n+1$ using the induction hypothesis (and the definition of $\operatorname{th}_{r}^{n+1}$ ).

5) We prove it by induction on $n$. The step from $n$ to $n+1$ is very straightforward. For $n=0$, we prove the statement by induction on $r$. For $r=0$ note $\operatorname{th}_{2^{r}}^{n}(\bar{a}, \mathfrak{B})=\operatorname{th}_{2^{0}}^{0}(\bar{a}, \mathfrak{B})=\operatorname{th}_{1}^{0}(\bar{a}, \mathfrak{B})$ so there is nothing to prove. For $r=r(0)+1$ just note that for $s_{0} \leq 2^{r}$ we have: $d(x, y) \leq s_{0}$ is equivalent to $\bigvee_{\substack{s_{1}+s_{2}=s_{0} \\ s_{1}, s_{2} \leq 2^{r(0)}}}(\exists z)\left[d(x, z) \leq s_{1} \& d(z, y) \leq s_{2}\right]$

6) The first phrase is a special case of the second (with $M_{i}$ a model with a single element: $i)$. Let $n(\ell) \stackrel{\text { def }}{=} n_{\ell}$. For the second phrase we prove the following more general statement by induction on $d$ :

$(*)_{d}$ Assume that for $\ell=1,2$ we have:

$$
M_{\ell}=\sum_{i<n(\ell)} M_{i}^{\ell} \text { and } 0 \leq i_{\ell}(1)<i_{\ell}(2)<\ldots<i_{\ell}\left(k^{*}-1\right)<n(\ell)
$$

we stipulate $i_{\ell}(0)=-1$ and $i_{\ell}\left(k^{*}\right)=n(\ell)$ (possibly $k^{*}=1$ ), assume further $\bar{a}_{k}^{\ell} \subseteq M_{i_{\ell}(k)}^{\ell}$ has length $m(k)$ for $k<k^{*}$. Also assume that for each $k=0, \ldots, k^{*}-1$ we have $\operatorname{th}^{d}\left(\bar{a}_{k}^{1}, M_{i_{1}(k)}^{1}\right)=\operatorname{th}^{d}\left(\bar{a}_{k}^{2}, M_{i_{2}(k)}^{2}\right)$ and $\left(i_{1}(k+1)-\right.$ $\left.i_{1}(k)-1\right)$ and $\left(i_{2}(k+1)-i_{2}(k)-1\right)$ are equal or both $\geq 2^{d}-1$. Lastly assume $\operatorname{th}^{d}\left(M_{i}^{1}\right)=\operatorname{th}^{d}\left(M_{j}^{2}\right)$ at least when $(\exists m)\left[i \in\left(i_{1}(m), i_{1}(m+1)\right) \& j \in\right.$ $\left(i_{2}(m), i_{2}(m+1)\right]$ (holds automatically when proving second phrase of $\left.(6)\right)$. $\underline{\text { Then }}$

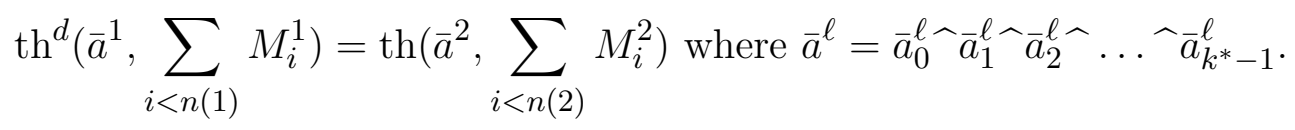

The proof is straightforward, and for the case $k^{*}=1$ we get the desired conclusions. Lastly the third phrase of $(6)$ is also a particular case of $(*)_{d}$ : let $n(1)=n(2)=k, k^{*}=n(\ell)+1, i_{\ell}(m)=m-1$, and $\bar{a}_{i}^{\ell}=\langle\rangle$.

7) The proof is like that of $(6)$, but in $(*)_{d}$ we add $\bigwedge_{k=1}^{k^{*}-1} i_{1}(k)=i_{2}(k)$.

Remark: If we will want to quantify on sequence of elements (i.e. use $\bar{c}$ rather than c) this helps. 
2.7A Claim: Let $\mathfrak{B}$ be a system, let $\left\langle a_{i}: i<m\right\rangle$ and $\left\langle r_{i}: i<m\right\rangle$ (where $a_{i} \in \mathfrak{B}$ and $r_{i} \in \mathbb{N}$ ) $f_{n}: \mathbb{N} \rightarrow \mathbb{N}$ for $n \in \mathbb{N}$ (not necessarily as in 2.5) be given

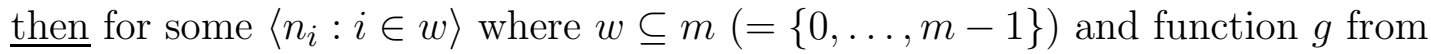
$\{0,1, \ldots, m-1\}$ to $w$ which is the identity on $w$ we have: $\sum_{i \in w} n_{i} \leq m-|w|$ and the sets in $\left\langle N_{f_{n_{i}}\left(\sum\left\{r_{j}: g(j)=i\right\}\right)}\left(a_{i}\right): i \in w\right\rangle$ are pairwise disjoint and $\bar{a}$ is included in their union provided that

(*) $2 f_{n_{1}}\left(r_{1}\right)+f_{n_{2}}\left(r_{2}\right) \leq f_{n_{1}+n_{2}+1}\left(r_{1}+r_{2}\right)$ and $f$ non decreasing in $r$ and in $n$ (considering $f$ a two place function from $\mathbb{N}$ to $\mathbb{N}$ ).

Proof: We call $\langle w, \bar{n}, g\rangle$ with $\bar{n}=\left\langle n_{i}: i \in w\right\rangle$ candidate if it satisfies all the requirements in the conclusion of $2.7 \mathrm{~A}$ except possibly the "pairwise disjoint". Clearly there is a candidate: $w=\{0, \ldots, m-1\}, \bigwedge_{i \in w} n_{i}=0, g$ the identity on $w$. So there is a candidate $\langle w, \bar{n}, g\rangle$ with $|w|$ minimal. If the disjointness demand holds, then we are done. So assume $i(1) \neq i(2)$ are in $w$ and there is $x$ belonging to $N_{f_{n_{i(1)}}^{+}}^{+}\left(\sum\left\{r_{j}: g(j)=i(1)\right\}\right)\left(a_{i(1)}\right)$ and to $N_{f_{n_{i(2)}}^{+}\left(\sum\left\{r_{j}: g(j)=i(2)\right\}\right.}\left(a_{i(1)}\right)$.

Let $w^{\prime}=w \backslash\{i(2)\}, g^{\prime}$ be a function with domain $\{0, \ldots, m-1\}$ defined by: $g^{\prime}(j)$ is $g(j)$ if $g(j) \neq i(2)$, and $g^{\prime}(j)$ is $i(1)$ if $g(j)=i(2)$. Lastly define $n_{i}^{\prime}$ for $i \in w^{\prime}: n_{i}^{\prime}=n_{i}$ if $i \neq i(1)$ and $n_{i}^{\prime}=n_{i(1)}+n_{i(2)}+1$ if $i=i(1)$ and let $\bar{n}^{\prime}=\left\langle n_{i}^{\prime}: i \in w^{\prime}\right\rangle$. Now we shall show below that $\left(w^{\prime}, \bar{m}^{\prime}, g^{\prime}\right)$ is a candidate thus finishing the proof: for this we have to check the two relevant conditions. First

$$
\begin{aligned}
& \sum_{i \in w^{\prime}} n_{i}^{\prime}=\sum_{\substack{i \in w^{\prime} \\
i \neq i(1)}} n_{i}^{\prime}+n_{i(1)}^{\prime}=\sum_{\substack{i \in w^{\prime} \\
i \neq i(1)}} n_{i}+n_{i(1)}+n_{i(2)}+1= \\
& \sum_{i \in w} n_{i}+1 \leq m-|w|+1=m-(|w|-1)=m-\left|w^{\prime}\right| .
\end{aligned}
$$

Secondly, why $\bigcup_{i \in w} N_{f_{n_{i}^{\prime}}^{+}\left(\sum\left\{r_{j}: g(j)=i\right\}\right)}\left(a_{i}\right)$ includes $\bar{a}$ ? if $j(*)<m$ then for some $i \in w$ we have $a_{j(*)} \in N_{f_{n_{i}}\left(\sum\left\{r_{j}: g(j)=i\right\}\right)}^{+}$; if $i \neq i(1), i(2)$ then $i \in w^{\prime}$, and

$$
N_{f_{n_{i}^{\prime}}^{+}\left(\sum\left\{r_{j}: g^{\prime}(j)=i\right\}\right)}\left(a_{i}\right)=N_{f_{n_{i}}\left(\sum\left\{r_{j}: g(j)=i\right\}\right)}^{+}\left(a_{i}\right)
$$

so we are done; if $i=i(1)$ then $n_{i}^{\prime}=n_{i(1)}+n_{i(2)}+1 \geq n_{i(1)}=n_{i}$ and

$$
\sum\left\{r_{j}: g^{\prime}(j)=i\right\} \geq \sum\left\{r_{j}: g(j)=i\right\}
$$

hence

$$
N_{f_{n_{i}^{\prime}}^{\prime}\left(\sum\left\{r_{j}: g^{\prime}(j)=i\right\}\right)}\left(a_{i}\right) \supseteq N_{f_{n_{i}}\left(\sum\left\{r_{j}: g(j)=i\right\}\right)}^{+}\left(a_{i}\right)
$$

and we are done. 
We are left with the case $i=i(2)$, so by the choice of $i$ we have $d\left(a_{j(*)}, a_{i(2)}\right) \leq$ $f_{n_{i(2)}}\left(\sum\left\{r_{j}: g(j)=i(2)\right\}\right)$ and by the choice of $x$ (and $\left.i(1), i(2)\right)$ above $d\left(a_{i(2)}, x\right) \leq f_{n_{i(2)}}\left(\sum\left\{r_{j}: g(j)=i(2)\right\}\right)$ and $d\left(x, a_{i(1)}\right) \leq f_{n_{i(1)}}\left(\sum\left\{r_{j}:\right.\right.$ $g(j)=i(1)\})$. So as $d$ is a metric (i.e. the triangular inequality) $d\left(a_{j(*)}, a_{i(1)}\right) \leq$ $2 f_{n_{i(2)}}\left(\sum\left\{r_{j}: g(j)=i(2)\right\}\right)+f_{n_{i(1)}}\left(\sum\left\{r_{j}: g(j)=i(1)\right\}\right)$. Now $\sum\left\{r_{j}: g^{\prime}(j)=\right.$ $i(1)\}=\sum\left\{r_{j}: g(j)=i(1)\right\}+\sum\left\{r_{j}: g(j)=i(2)\right\}$ (by the definition of $g^{\prime}$ ) and $n_{i(1)}^{\prime}=n_{i(1)}+n_{i(2)}+1$, hence what we need is

(*) $2 f_{n^{1}}\left(r^{1}\right)+f_{n^{2}}\left(r^{2}\right) \leq f_{n^{1}+n^{2}+1}\left(r^{1}+r^{2}\right)$

which is assumed.

2.7B Remark: 1) We can replace in $2.7 \mathrm{~A}$ and its proof $\sum\left\{r_{j}: g(j)=i\right\}$ by $\max \left\{r_{j}: g(j)=i\right\}$ and $($ in $(*)) r^{1}+r^{2}$ by $\max \left\{r^{1}, r^{2}\right\}$.

(2) Concerning $(*)$, letting $n=\max \left\{n^{1}, n^{2}\right\}$ and $r=\max \left\{r^{1}, r^{2}\right\}$ it suffices to have

$(*)_{1} f_{n}(r)$ is non decreasing in $n$ and in $\mathrm{r}$.

$(*)_{2} 3 f_{n}(r) \leq f_{n+1}(r)$

2.8 Definition: 1) For a system $\mathfrak{B}$, and $r, n, m \in \mathbb{N}$, and $\bar{a}=\left\langle a_{0}, \ldots, a_{m-1}\right\rangle \subseteq \mathfrak{B}$ we call $\bar{a}$ a $(\mathfrak{B}, r)$-component if $\bar{a} \subseteq N_{r}^{+}\left(a_{0}\right)$. In this case we define $\operatorname{bth}_{r}^{n}(\bar{a}, \mathfrak{B})$ (bth is for bounded theory). We do it by induction on $n$ (for all $r$ ) (the function $f$ from 2.5 is an implicit parameter.)

$(\alpha) \operatorname{bth}_{r}^{0}(\bar{a}, \mathfrak{B})=\operatorname{th}^{0}\left(\bar{a}, \mathfrak{B}_{r}\right)$

$(\beta) \operatorname{bth}_{r}^{n+1}(\bar{a}, \mathfrak{B})$ is $\left\langle\mathbf{t}_{0}, \mathbf{t}_{1}, \mathbf{t}_{2}\right\rangle$ where

$$
\begin{aligned}
\text { (i) } \mathbf{t}_{0} & =\operatorname{bth}_{r}^{n}(\bar{a}, \mathfrak{B}) \\
\text { (ii) } \mathbf{t}_{1} & =\left\{\operatorname{bth}_{r}^{n}(c, \mathfrak{B}): c \in N_{f_{n}^{(2)}(r)}^{+}\left(a_{0}\right) \backslash N_{f_{n}(r)}^{+}\left(a_{0}\right)\right\} \\
\text { (iii) } \mathbf{t}_{2} & =\left\{\operatorname{bth}_{f_{n}^{(2)}(r)}^{n}\left(\bar{a}^{\wedge}\langle c\rangle, \mathfrak{B}\right): c \in N_{f_{n}(r)}^{+}\left(a_{0}\right)\right\}
\end{aligned}
$$

2) If $\sigma$ is a vocabulary of systems and $r, n, m \in \mathbb{N}$ then $\operatorname{BTH}_{r}^{n}(m, \sigma)$ is the set of formally possible $\operatorname{bth}_{r}^{n}(\bar{a}, \mathfrak{B}),\left(\mathfrak{B}\right.$ a $\sigma$-system, $\bar{a}=\left\langle a_{0}, \ldots, a_{m-1}\right\rangle \subseteq \mathfrak{B}$ and $\bar{a}$ is a $(\mathfrak{B}, r)$-component).

2.9 Claim: 1) For any $\sigma$ (vocabulary of systems), numbers $n, m, r \in \mathbb{N}$, and $\mathbf{t} \in$ $\operatorname{BTH}_{r}^{n}(m, \sigma)$, there is $\varphi=\varphi\left(x_{0}, \ldots, x_{m-1}\right) \in \mathcal{L}_{\sigma}\left(\right.$ even $\left.\mathcal{L}_{\sigma\left(\mathfrak{B}_{f_{n}(r)}\right)}\right)$ of quantifier depth $n$ such that for any $\sigma$-system $\mathfrak{B}$, and $\bar{a}=\left\langle a_{0}, \ldots, a_{m-1}\right\rangle \subseteq \mathfrak{B}$ a $(\mathfrak{B}, r)$ component we have:

$$
\mathbf{t}=\operatorname{bth}_{r}^{n}(\bar{a}, \mathfrak{B}) \quad \text { iff } \mathfrak{B}_{f_{n}(r)}\left\lceil N_{f_{n}(r)}^{+}\left(a_{0}\right) \models \varphi[\bar{a}] .\right.
$$


2) If $n \geq m, \bar{b}$ is the permutation of $\bar{a}$ by the function $h$ or $\bar{b}=\left\langle a_{\ell}: \ell<k\right\rangle$ for some $k \leq \ell g(\bar{a})$ and $b_{0}=a_{0}$ then from $\mathbf{t}=\operatorname{bth}_{r}^{n}(\bar{a}, \mathfrak{B})$ we can compute $\operatorname{bth}_{r}^{m}(\bar{b}, \mathfrak{B})$ (using $n, m, r$, and $h$ or $k$ ).

Proof: Should be clear (see convention 2.5(1)).

Remark: Concerning 2.9(2) we can say something also in the case $b_{0} \neq a_{0}$ but there was no real need.

2.10 Definition: For a $\sigma$-system $\mathfrak{B}$, and $r, n, m \in \mathbb{N}$ we define $\mathfrak{B}_{r, m}^{n}$ as the expansion of $\mathfrak{B}$ by the relations $R_{\mathbf{t}}^{\ell}=\left\{\bar{a}: \bar{a}\right.$ is a $\left(\mathfrak{B}, r^{\prime}\right)$-component , $\mathbf{t}=$ $\operatorname{bth}_{r^{\prime}}^{n}(\bar{a}, \mathfrak{B}),[\ell=1 \Rightarrow \bar{a} \subseteq M]$ and $\left.[\ell=2 \Rightarrow \bar{a} \subseteq I]\right\}$ for each $\mathbf{t} \in \mathrm{BTH}_{r^{\prime}}^{n}\left(m^{\prime}, \sigma\right)$ $m^{\prime} \leq m+n, r^{\prime} \leq f_{n}^{(3)}(r)$ and $\ell \in\{1,2\}$. We let $I_{r, m}^{n}[\mathfrak{B}]=\mathfrak{B}_{r, m}^{n}\lceil I$. Writing $\bar{r}=\left\langle r_{\ell}: \ell<k\right\rangle$ we mean $\max (\bar{r})$. Writing $\mathfrak{B}_{\bar{r}, \bar{m}}^{n}$ means the common expansion of $\mathfrak{B}_{r_{\ell}, m_{\ell}}^{n}$ for $\ell<\ell g(\bar{r})=\ell g(\bar{m})$ if $\ell g(\bar{r})=0$ we mean $\mathfrak{B}_{0,0}^{n}$ (we could alternatively use $\mathfrak{B}_{\max (\bar{r}), \max (\bar{m})}^{n}$, make little difference). Writing $\leq r$ we mean for every $r^{\prime} \leq r$

2.11 Claim: $I_{\bar{r}, \bar{m}}^{n+1}[\mathfrak{B}]$ essentially expands $I_{\bar{r}^{\prime}, \bar{m}^{\prime}}^{n}[\mathfrak{B}]$ when

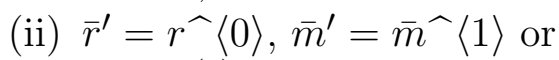

(ii) $\bar{r}^{\prime} \leq f_{n}^{(2)}(\bar{r}), \bar{m}^{\prime} \leq\left\langle m_{i}+1: i<\lg (\bar{m})\right.$

(essentially expand means that every predicate in the latter is equivalent to a quantifier free formula in the former, the function giving this is the scheme of expansion).

Proof: Should be clear by $(i)$ of $(\beta)$ of $2.8(1)$.

2.12 Definition: For a system $\mathfrak{B}$ and $n, m \in \mathbb{N}$ and $\bar{r}=\left\langle r_{\ell}: \ell<m\right\rangle$ such that $r_{\ell} \in \mathbb{N}$ and $\bar{a}=\left\langle a_{0}, \ldots, a_{k-1}\right\rangle \subseteq I^{\mathfrak{B}}$

1) We say $\bar{a}$ is $(n, \bar{r})$-sparse for $\mathfrak{B}$ if $\bar{r}=\left\langle r_{\ell}: \ell<m\right\rangle$ and

$$
\ell<k<m \text { and } N_{f_{n}\left(r_{\ell}\right)}^{+}\left(a_{\ell}\right) \cap N_{f_{n}\left(r_{k}\right)}^{+}\left(a_{k}\right)=\emptyset
$$

moreover (slightly stronger)

$$
d\left(a_{\ell}, a_{k}\right) \geq f_{n}\left(r_{\ell}\right)+f_{n}\left(r_{k}\right)+1 .
$$


2) We define $\operatorname{uth}_{\bar{r}}^{n}(\bar{a}, \mathfrak{B})$ for an $(n, \bar{r})$-sparse $\bar{a} \subseteq \mathfrak{B}$, by induction on $n$ : $\operatorname{uth}_{\bar{r}}^{0}(\bar{a}, \mathfrak{B})=\operatorname{th}^{0}(\bar{a}, \mathfrak{B})$ and $\operatorname{uth}_{\bar{r}}^{n+1}(\bar{a}, \mathfrak{B})=\left\langle\mathbf{t}_{0}, \mathbf{t}_{1}\right\rangle$ where:

$$
\mathbf{t}_{0}=\left\{\left\langle\bar{s}, \operatorname{uth}_{\bar{s}}^{n}(\bar{a}, \mathfrak{B})\right\rangle: \bar{s} \leq f_{n}^{(2)}(r)\right\}
$$

$\left(\right.$ see $2.5, f_{n}^{(2)}(\bar{r})=\left\langle f_{n}\left(f_{n}\left(r_{\ell}\right)\right): \ell<\ell g(\bar{a})\right\rangle$, remember $\left.f_{n}^{(2)}(\bar{r}) \leq f_{n+1}(\bar{r})\right)$

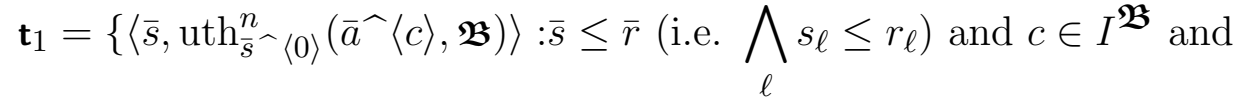

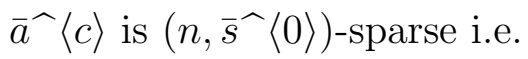

$$
\begin{aligned}
& \left.N_{f_{n}(0)}^{+}(c) \text { is disjoint from } N_{f_{n}\left(s_{\ell}\right)}^{+}\left(a_{\ell}\right) \text { for } \ell<\ell g(\bar{a})\right\} \text {. }
\end{aligned}
$$

3) $\operatorname{UTH}_{\bar{r}}^{n}(m, \sigma)$ is the set of formally possible $\operatorname{uth}_{\bar{r}}^{n}(\bar{a}, \mathfrak{B})(\bar{a}$ is $(n, \bar{r})$-sparse for $\mathfrak{B}$ of length $m, \mathfrak{B}$ a $\sigma$-system, etc.).

2.13 Claim: 1) For every $\mathbf{t} \in \operatorname{UTH}_{r}^{n}(m, \sigma)$ there is a formula $\varphi=\varphi\left(x_{0}, \ldots, x_{k-1}\right) \in$ $\mathcal{L}_{\sigma_{f^{*}(\bar{r})}}$ where $f_{n}^{*}(\bar{r})=\min \left\{m:\right.$ if $\ell_{1}<\ell_{2}<\ell g(\bar{a})$ then $\left.m>f_{n}\left(r_{\ell_{1}}\right)+f_{n}\left(r_{\ell_{2}}\right)\right\}$ of quantifier depth $n$ such that: For a $\sigma$-system $\mathfrak{B}$ and $(n, \bar{r})$-sparse $\bar{a}=$ $\left\langle a_{0}, \ldots, a_{m-1}\right\rangle \subseteq I^{\mathfrak{B}}$, we have: $\mathfrak{B} \models \varphi\left[a_{0}, \ldots, a_{m-1}\right]$ iff $\mathbf{t}=\operatorname{uth}_{\bar{r}}^{n}(\bar{a}, \mathfrak{B})$ (and being $(n, \bar{r})$-sparse is equivalent to some quantifier free formula).

2) In Definition 2.12 only $\mathfrak{B}\lceil I$ matters and in part (1), the quantifications are on $I$ only.

3) If $\mathfrak{B}^{\prime}$ essentially expands $\mathfrak{B}$ then from (the scheme of expansion and) $\operatorname{uth}_{\bar{r}}^{n}\left(\bar{a}, \mathfrak{B}^{\prime}\right)$ we can compute $\operatorname{uth}_{\bar{r}}^{n}(\bar{a}, \mathfrak{B})$.

Proof: Should be clear.

2.14 Main Lemma: Let $\sigma$ be a system-vocabulary; if $\otimes_{0}$ holds then $\otimes_{n}$ holds for every $n$ where:

$\otimes_{n}$ there are functions $F_{n, \bar{r}, \bar{m}}$, for $\bar{r}=\left\langle r_{\ell}: \ell<k\right\rangle, \bar{m}=\left\langle m_{\ell}: \ell<k\right\rangle$, where $k, r_{\ell}, m_{\ell} \in \mathbb{N}$, such that:

$(*)$ if $\mathfrak{B}$ is a $\sigma$-system, $\bar{a}=\bar{a}^{0 \curlywedge} \ldots \widehat{a}^{k-1}, \bar{a}^{\ell}$ an $\left(\mathfrak{B}, r_{\ell}\right)$-component, $\left\langle a_{0}^{\ell}: \ell<\right.$ $k\rangle$ is $(n, \bar{r})$-sparse and $\bar{m}=\left\langle\ell g\left(\bar{a}^{\ell}\right): \ell<k\right\rangle \underline{\text { then }}$ letting $\mathbf{t}_{\ell} \stackrel{\text { def }}{=} \operatorname{bth}_{r_{\ell}}^{n}\left(\bar{a}^{\ell}, \mathfrak{B}\right)$ (for $\ell<k$ ) and $\mathbf{t} \stackrel{\text { def }}{=} \operatorname{uth}_{\bar{r}}^{n}\left(\left\langle h\left(a_{0}^{\ell}\right): \ell<k\right\rangle, I_{\bar{r}, \bar{m}}^{n}[\mathbf{B}]\right)$ we have $\operatorname{th}_{0}^{n}(\bar{a}, \mathfrak{B})=$ $F_{n, \bar{r}, \bar{m}}\left(\mathbf{t}, \mathbf{t}_{0}, \ldots, \mathbf{t}_{k-1}\right)$.

$(* *) F_{n, \bar{r}, \bar{m}}$ is recursive in its variables, $n, \bar{r}, \bar{m}$ and the functions $F_{0, \bar{r}^{\prime}, \bar{m}^{\prime}}$ where $\bar{m}^{\prime}=\left\langle m_{i}^{\prime}: i<k^{\prime}\right\rangle, k^{\prime} \geq k, \bar{r}^{\prime} \leq f_{n}^{*}(\bar{r})$ (see 2.13(1)) and for $i<k$ we have $m_{i}^{\prime} \geq m_{i}$ and $\sum_{i<k}\left(m_{i}^{\prime}-m_{i}\right)+\sum_{i=k}^{k^{\prime}-1} m_{i}^{\prime} \leq n$. 
2.14A Remark: Why we use $\operatorname{th}_{0}^{n}$ and not $\operatorname{th}_{r}^{n}$ (in the conclusion of $\left.(*)\right)$ ? We can if we assume " $d(x, y) \leq s \& x \in I \& y \in I$ " is an atomic formula for $\mathfrak{B}$ for $s \leq r$.

Proof: We prove this by induction on $n$, (for all $\bar{r}, \bar{m})$, so for $n=0$ clearly $\otimes_{n}$ holds. So assume $\otimes_{n}$ and we shall prove $\otimes_{n+1}$. We shall now describe a value $\mathbf{t}$ computed from $\operatorname{bth}_{r_{\ell}}^{n+1}\left(\bar{a}^{\ell}, \mathfrak{B}\right)($ for $\ell<k)$ and $\operatorname{uth}^{n+1}\left(\left\langle h\left(a_{0}^{\ell}\right): \ell<k\right\rangle, I_{\bar{r}, \bar{m}}^{n+1}[\mathfrak{B}]\right)$. Our intention is that $\mathbf{t}=\operatorname{th}^{n+1}(\bar{a}, \mathfrak{B})$. Remember $\mathbf{t}=\left\{\operatorname{th}^{n}(\bar{a} \widehat{\langle}\langle c\rangle, \mathfrak{B}): c \in \mathfrak{B}\right\}$.

Now $\mathbf{t}$ will be the union of two sets.

We use an informal description as it is clearer.

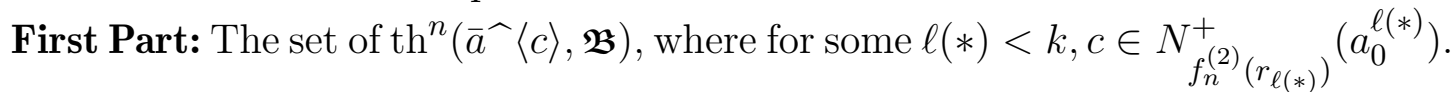
Why can we compute this (using the induction hypothesis of course), for each such $c$ we have: let $\bar{b}^{\ell}=\bar{a}^{\ell}$ if $\ell<k, \ell \neq \ell(*)$ and let $\bar{b}^{\ell(*)}=\bar{a}^{(\ell(*))}\langle c\rangle$ (i.e. $\left.b_{m_{\ell(*)}^{\ell(*)}}=c\right)$; next $r_{\ell}^{\prime}$ is: $r_{\ell}$ for $\ell \neq \ell(*)$ and $f_{n}^{(2)}\left(r_{\ell(*)}\right)$ if $\ell=\ell(*)$. Necessarily for $\ell<k, m_{\ell}^{i}$ is $m_{\ell}$ if $\ell \neq \ell(*)$ and $m_{\ell(*)}+1$ if $\ell=\ell(*)$. Now:

( $\alpha) N_{f_{n}\left(r_{\ell}^{\prime}\right)}^{+}\left(b_{0}^{\ell}\right)$ for $\ell<k$ are pairwise disjoint, moreover for $\ell(1)<\ell(2)<k$, $d\left(b_{0}^{\ell(1)}, b_{0}^{\ell(2)}\right)>f_{n}\left(r_{\ell(1)}^{\prime}\right)+f_{n}\left(r_{\ell(2)}^{\prime}\right)$.

[Why? for $\ell=\ell(*)$ remember that for every $r$ : $\left.f_{n}\left(f_{n}^{(2)}(r)\right)=f_{n}^{(3)}(r)\right) \leq$ $f_{n+1}(r)$ and for $\ell \neq \ell(*)$ remember that for every $r: f_{n}(r) \leq f_{n+1}(r)$ so in all cases clearly $f_{n}\left(r_{\ell}^{\prime}\right) \leq f_{n+1}\left(r_{\ell}\right)$ and $b_{0}^{\ell}=a_{0}^{\ell}$. So if $\ell(1) \neq \ell(2)$ are $<k, N_{f_{n}\left(r_{\ell(1)}^{1}\right)}^{+}\left(a_{0}^{\ell(1)}\right) \cap N_{f_{n}\left(r_{\ell(2)}^{\prime}\right)}^{+}\left(b_{0}^{\ell(2)}\right)=\emptyset$ as: if $\ell(1) \neq \ell(*), \ell(2) \neq \ell(*)$, trivial. Otherwise without loss of generality $\ell(2)=\ell(*)$, as $\bar{a}$ satisfies the assumption of $(*)((*)$ is from the lemma) $\bar{a}$ is $(n+1, \bar{r})$-sparse hence (see Def. 2.12(1)) $d\left(a_{0}^{\ell(1)}, a_{0}^{\ell(*)}\right)>f_{n+1}\left(r_{\ell(1)}\right)+f_{n+1}\left(r_{\ell(2)}\right)$, hence $d\left(b_{0}^{\ell(1)}, b_{0}^{\ell(*)}\right)=$ $d\left(a_{0}^{\ell(1)}, a_{0}^{\ell(*)}\right)>f_{n+1}\left(r_{\ell(1)}\right)+f_{n+1}\left(r_{\ell(2)}\right) \geq f_{n}\left(r_{\ell(1)}\right)+f_{n}\left(f_{n}^{(2)}\left(r_{\ell(*)}\right)\right)=$ $\left.f_{n}\left(r_{\ell(1)}^{\prime}\right)+f_{n}\left(r_{\ell(*)}^{\prime}\right)\right)$, as required.]

(ß) $\bar{b}^{\ell} \subseteq N_{r_{\ell}^{\prime}}^{+}\left(b_{0}^{\ell}\right)$, so $\bar{b}^{\ell}$ is an $r_{\ell^{-c o m p o n e n t . ~}}^{\prime}$

[Why? when $\ell=\ell(*)$ as $r_{\ell} \leq f_{n}^{(2)}\left(r_{\ell}\right)=r_{\ell}^{\prime}$ and assumption on $c$; for $\ell \neq \ell(*)$ trivial.]

$(\gamma)$ We can compute $\operatorname{bth}_{r_{\ell}^{\prime}}^{n}\left(\bar{b}^{\ell}, \mathfrak{B}\right)$ for $\ell \neq \ell(*)$

[Why? by monotonicity properties of bth i.e. by $2.9(2)$ (that is clause (i) i.e.

$\mathbf{t}_{0}$ from Def. 2.8(1)( $\left.\left.\beta\right)\right)$ ];

$(\delta)$ We can compute the set of possibilities of $\operatorname{bth}_{r_{\ell(*)}^{\prime}}^{n}\left(\bar{b}^{\ell(*)}, \mathfrak{B}\right)$.

[Why? those possibilities are listed in bth $^{n+1}$ (see $\mathbf{t}_{2}$ in Definition $2.8(1)(\beta)$ mainly clause(ii)).] 


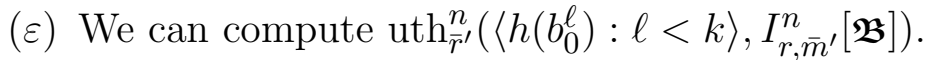

[As we can compute $\operatorname{uth}_{\bar{s}}^{n}\left(\left\langle h\left(b_{0}^{\ell}\right): \ell<k\right\rangle, I_{\bar{r}, \bar{m}}^{n+1}[\mathfrak{B}]\right)$ for $\bar{s} \leq f_{n}^{(2)}(\bar{r})$ by the definition of $\mathbf{t}_{0}$ in Def. 2.12(2), choose $\bar{s}=\bar{r}^{\prime}$; now, by $2.11, I_{\bar{r}, \bar{m}}^{n+1}[\mathfrak{B}]$ essentially expand $I_{\bar{r}^{\prime}, \bar{m}^{\prime}}^{n}[\mathfrak{B}]$ (see clause (ii) there) hence by $2.13(3)$ we can get the required object.]

( $\zeta$ ) We can compute the set of possibilities of $\operatorname{th}^{n}\left(\bar{b}^{0 \wedge} \ldots{ }^{\wedge} \bar{b}^{k-1}, \mathfrak{B}\right)$ gotten as

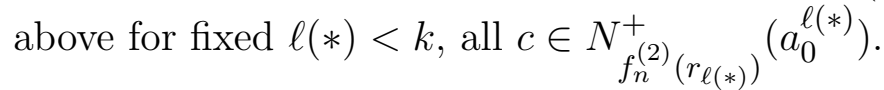

[Why? by $(\alpha)-(\varepsilon)$ above and $\oplus_{n}$.]

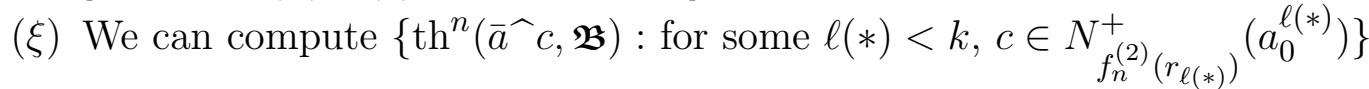

Second Part: The set of $\operatorname{th}^{n}(\bar{a} \widehat{\sim}\langle c\rangle, \mathfrak{B})$ where for each $\ell<k, c \notin N_{f_{n}^{(2)}(r)+f_{n}(0)}^{+}\left(a_{0}^{\ell}\right)$.

Why can we compute this? for such a $c$ we can let $k^{\prime}=k+1, \bar{a}^{k}=\langle c\rangle$ (so $\left.c=a_{0}^{k}, m_{k}=1\right)$. Let $r_{\ell}^{\prime}=r_{\ell}$, (for $\ell<k$ ) and $r_{k}^{\prime}=0$. Let $\bar{m}^{\prime}$ be $\bar{m}^{\wedge}\langle 1\rangle$. Now

$(\alpha) N_{f_{n}\left(r_{\ell}\right)}^{+}\left(a_{0}^{\ell}\right)$ for $\ell \leq k$ are pairwise disjoint and $d\left(a_{0}^{\ell(1)}, a_{0}^{\ell(2)}\right)>f_{n}\left(r_{\ell(1)}\right)+$ $f_{n}\left(r_{\ell(2)}\right)$.

[Why? as $f_{n}(r) \leq f_{n+1}(r)$ for $\ell(1)<\ell(2)<k$ this is trivial, and for $\ell(1)=\ell<k=\ell(2)$ we have $d\left(a_{0}^{\ell(1)}, a_{0}^{\ell(2)}\right)=d\left(a_{0}^{\ell}, a_{0}^{k}\right)=d\left(a_{0}^{\ell}, c\right)>f_{n}^{(2)}\left(r_{\ell}\right) \geq$ $f_{n}\left(r_{\ell}\right)+f_{n}(0)=f_{n}\left(r_{\ell}^{\prime}\right)+f_{n}\left(r_{k}^{\prime}\right)$, as required].

(ß) $\bar{a}^{\ell} \subseteq N_{r_{\ell}^{\prime}}^{+}\left(a_{0}^{\ell}\right)$, so $\bar{a}^{\ell}$ is an $r_{\ell^{-c o m p o n e n t . ~}}^{\prime}$

[Why? for $\ell<k$ as $r_{\ell}^{\prime}=r_{\ell}$, for $\ell=k$ as $\left.\bar{a}^{k}=\left\langle a_{0}^{k}\right\rangle(=\langle c\rangle)\right]$.

$(\gamma)$ we can compute $\operatorname{bth}_{r_{\ell}^{\prime}}^{n}\left(\bar{a}^{\ell}, \mathfrak{B}\right)$ for $\ell<k$

[Why? by monotonicity properties of bth i.e. 2.9(2)].

$(\delta)$ we can compute the possibilities for pairs $\left(\mathbf{t}^{\prime}, \mathbf{t}^{\prime \prime}\right)$ where $\mathbf{t}^{\prime}=\operatorname{bth}_{0}^{n+1}(\langle c\rangle, \mathfrak{B})$ and $\mathbf{t}^{\prime \prime}=\operatorname{uth}_{\bar{r}^{\prime}}^{n}\left(\left\langle h\left(a_{0}^{\ell}\right): \ell<k\right\rangle \uparrow\langle h(c)\rangle, I_{\bar{r}, \bar{m}}^{n+1}[\mathfrak{B}]\right)$.

[Why? straightforward; by the definition of $\mathrm{uth}^{n+1}$, i.e. $\mathbf{t}_{1}$ of Def. 2.12(2) and $\left.I_{\bar{r}, \bar{m}^{\prime}}^{n}[\mathfrak{B}]\right]$.

$(\varepsilon)$ in $(\delta)$ we can replace $I_{\bar{r}, \bar{m}}^{n+1}[\mathfrak{B}]$ by $I_{\bar{r}^{\prime}, \bar{m}^{\prime}}^{n}[\mathfrak{B}]$.

[Why? by 2.11 and 2.13(3).]

By the induction hypothesis this is enough.

Why the union of the two parts is $\operatorname{th}^{n}(\bar{a}, \mathfrak{B})$ ?

Both obviously give subsets, and if $c$ fails the first part then $\ell<k \Rightarrow c \notin$ $N_{f_{n}^{(2)}\left(r_{\ell}\right)}^{+}\left(a_{0}^{\ell}\right)$. So $N_{f_{n}(0)}^{+}(c)$ is disjoint to such $N_{f_{n}\left(r_{\ell}\right)}^{+}\left(a_{0}^{\ell}\right)$ and moreover $d\left(a_{0}^{\ell}, c\right)>$ $f_{n}\left(r_{\ell}\right)+f_{n}(0)$. 
2.15 Conclusion: For any system vocabulary $\sigma$, and (first order) sentence $\varphi$ of quantifier depth $n$, given $F_{0, \bar{r}, \bar{m}}$ 's satisfying $\otimes_{0}$ of 2.14 we can compute numbers $r, m$ and a sentence $\psi_{\varphi}$ of quantifier depth $n$, (whose vocabulary is that of $\left.I_{r, m}^{n}[\mathfrak{B}]\right)$ such that:

$(*)$ if $\mathfrak{B}$ is a $\sigma$-system which satisfied $\otimes_{0}$ as exemplified by $\left\langle F_{0, \bar{r}, \bar{m}}: \bar{r}, \bar{m}\right\rangle \underline{\text { then }}$ $\mathfrak{B} \models \varphi \Leftrightarrow I_{r, m}^{n}[\mathfrak{B}] \models \psi_{\varphi}$.

Proof: By 2.14 and 2.13(1) and 2.7(1) (and see 2.14A(2)).

2.16 Conclusion: Let $\tau$ be a vocabulary (finite for simplicity) including a binary relation $<$, and $\varphi \in \mathcal{L}_{\tau}$. Then we can compute an $m<\omega$, formulas $\varphi_{i}(x) \in \mathcal{L}_{\tau}$ for $i<m$ with $d\left(\varphi_{i}\right) \leq d(\varphi)$ and a sentence $\psi_{\varphi} \in \mathcal{L}_{\tau_{1}^{*}}$ with $d\left(\psi_{\varphi}\right) \leq d(\varphi)$ where $\tau_{1}^{*} \stackrel{\text { def }}{=}\{<\} \cup\left\{P_{i}: i<m\right\}$ ( $m \in \mathbb{N}$ computable from $\varphi$, each $P_{i}$ a unary predicate) satisfying the following:

(*) Assume $M$ is a finite $\tau$-model, $<^{M}$ a linear order, $P \in \tau$ is unary, such that: if $R \in \tau \backslash\{<\}$ and $\bar{a}=\left\langle a_{0}, \ldots, a_{n(R)-1}\right\rangle \in R^{M}$, then $P^{M} \cap[\min \bar{a}, \max \bar{a}]_{M}$ has at most one member.

Define $I[M]=\left(P^{M}, \ldots, P_{\ell} \ldots\right)_{\ell<m}$ where $P_{\ell}=\left\{a \in P^{M}: \varphi_{\ell}[a]\right.$ is satisfied in $M \uparrow\left\{x: x \leq a,\left|[x, a]_{M} \cap P^{M}\right| \leq 3^{d[\varphi]}\right.$ or $\left.\left.a \leq x,\left|[a, x]_{M} \cap P^{M}\right| \leq 3^{d[\varphi]}\right\}\right\}$ $(d[\varphi]$ in the quantifier depth of $\varphi)$.

Then $M \models \varphi \Leftrightarrow I[M] \models \psi_{\varphi}$.

Proof: Should be clear from 2.15.

Remark: Concerning 2.16 we can deduce it also from $\S 4$.

\section{$\S 3$ Proof of the Main Theorem}

3.1 Definition: 1) For a finite set $J \subseteq I$ let $\operatorname{spr}(I, J)$ be the set of pairs $\left(Q^{\text {no }}, Q^{\text {yes }}\right)$, where $Q^{\text {no }} \subseteq I, Q^{\text {yes }} \subseteq I, Q^{\text {no }} \backslash J=Q^{\text {yes }} \backslash J,\left|Q^{\text {yes }} \Delta Q^{\text {no }}\right|=1(A \Delta B$ is the symmetric difference). Let $\operatorname{spr}(I)=\operatorname{spr}(I, I)$.

2) For finite $J \subseteq I$ let $\mu^{*}(I, J)$ be the following distribution on $\operatorname{spr}(I, J)$; it is enough to describe a drawing:

first choose $Q^{\text {no }} \subseteq I$ (all possibilities with probability $1 / 2^{|I|}$ )

then choose $s \in J$ (all possibilities with probability $1 /|J|$ )

finally let

$$
Q^{\text {yes }}= \begin{cases}Q^{\text {no }} \cup\{s\} & \text { if } s \notin Q^{\text {no }} \\ Q^{\text {no }} \backslash\{s\} & \text { if } s \in Q^{\text {no }}\end{cases}
$$


We write $\mu^{*}(I)$ for $\mu^{*}(I, I)$.

3.1A Remark: Note that the distribution $\mu^{*}(I, J)$ is symmetric for $Q^{\text {yes }}, Q^{\text {no }}$.

3.2 Definition: 1) For a linear order $I$ and $J \subseteq I$ let $\operatorname{npr}(I, J)=\left\{\left(Q^{\text {no }}, Q^{\text {yes }}\right)\right.$ : $Q^{\text {no }} \subseteq Q^{\text {yes }} \subseteq I$ and $Q^{\text {no }} \backslash J=Q^{\text {yes }} \backslash J$ and $\left.\left|Q^{\text {yes }} \backslash Q^{\text {no }}\right|=1\right\}$.

2) If $I$ is a set of natural numbers we use the usual order.

3) If $J=I$ we write $\operatorname{npr}(I)$.

4) Any $(I, J)$ is isomorphic to some $\left(n, J^{\prime}\right)$ so we can use such pairs above.

5) Let $\mu^{* *}(I, J)$-be the distributions $\mu^{*}(I, J)$ restricted to the case $Q^{\text {no }} \subseteq Q^{\text {yes }}$ (i.e. to $\operatorname{npr}(I, J))$.

3.3 Claim: Let $m, d \in \mathbb{N}$ be given, $\tau=\{<\} \cup\left\{P_{i}: i<m\right\}, P_{i}$ a unary predicate, $K=\left\{M: M\right.$ a $\tau$-model, $<^{M}$ a linear order $\}$.

1) For every $\varepsilon \in \mathbb{R}^{+}$for every $k$ large enough, for the distribution $\mu^{*}(k)$ on $\operatorname{spr}(k)=\left\{\left(Q^{\text {no }}, Q^{\text {yes }}\right): Q^{\text {no }}, Q^{\text {yes }} \subseteq k,\left|Q^{\text {yes }} \Delta Q^{\text {no }}\right|=1\right\}$ we have: if $M_{i}^{\mathfrak{t}} \in K$, for $i<k, \mathfrak{t} \in\{$ yes no $\}$, and we choose $\mu^{*}(k)$-randomly $\left(Q^{\text {no }}, Q^{\text {yes }}\right) \in \operatorname{spr}(k)$, then (*) the probability of $\operatorname{th}^{d}\left(\sum_{i<k} M_{i}^{\text {if }\left(i \in Q^{\text {yes }}\right)}\right)=\operatorname{th}^{d}\left(\sum_{i<k} M_{i}^{\text {if }\left(i \in Q^{\text {no }}\right)}\right)$ is at least $1-\varepsilon\left(\mathrm{th}^{d}\right.$ is defined as in 2.6(1) considering a model as a system by 2.2(4)). 2) Also if we first choose $\left(Q_{u}^{\text {no }}, Q_{u}^{\text {yes }}\right) \in \operatorname{spr}\left(\left[\left[\frac{k+1}{2}\right], k\right)\right)$ as above and then (possibly depending on the result) make a decision on a choice of $Q_{d}^{\text {no }}=Q_{d}^{\text {yes }} \subseteq\left[0,\left[\frac{k+1}{2}\right]\right)$ and let $Q^{\text {yes }}=Q_{d}^{\text {yes }} \cup Q_{u}^{\text {yes }}, Q^{\text {no }}=Q_{d}^{\text {no }} \cup Q_{u}^{\text {no }} \underline{\text { then }}(*)$ above still holds.

3) If we choose $Q^{\text {yes }} \subseteq k$ such that $\left|Q^{\text {yes }}\right|=\left[\frac{k+1}{2}\right]$ and then $Q^{\text {no }} \subseteq Q^{\text {yes }},\left|Q^{\text {no }}\right|+1=$ $\left|Q^{\text {yes }}\right|$ (all possibilities with the same probabilities) then with probability tending to 1 with $k$ going to $\infty$ we get that $(*)$ (of 3.3(1) above) holds.

4) The parallel of $(1)$ holds for $\mu^{* *}(k), \operatorname{npr}(k)$.

Before proving 3.3 we define and note:

3.4 Definition: For the $\tau, K, d$ from 3.3 , let $\zeta_{k}$ be the maximal real in $[0,1]$ such that: if $M_{i}^{\mathfrak{t}} \in K$ for $i<r, \mathfrak{t} \in\{$ yes, no $\}$ (where $r \in \mathbb{N}$ ), $J \supseteq\left\{i<r: \operatorname{th}^{d}\left(M_{i}^{\text {no }}\right) \neq\right.$ $\left.\operatorname{th}^{d}\left(M_{i}^{\mathrm{yes}}\right)\right\}$ has $k$ elements (and $J \subseteq I=r=\{0, \ldots, r-1\}$ ), then $\frac{k}{r}(1-$ $\left.\zeta_{k}\right) \geq \operatorname{Prob}_{\mu^{*}(I, J)}\left(\operatorname{th}^{d}\left(\sum_{i<r} M_{i}^{\mathrm{if}\left(i \in Q^{\mathrm{no}}\right)}\right) \neq \operatorname{th}^{d}\left(\sum_{i<r} M_{i}^{\mathrm{if}\left(i \in Q^{\mathrm{yes}}\right)}\right) \mid\left(Q^{\text {no }}, Q^{\text {yes }}\right) \in\right.$ $\operatorname{spr}(I, J))$. Let $\xi_{k}=1-\zeta_{k}$.

3.4A Observation: 1) $\zeta_{k}$ is well defined; and $\zeta_{k} \leq \zeta_{k+1}$

2) An alternative definition of $\zeta_{k}$ is that it is the maximum real in $[0,1]$ satisfying: $\left(1-\zeta_{k}\right)$ is not smaller than the relative-probability of $\operatorname{th}^{d}\left(\sum_{i<r} M_{i}^{\text {if }\left(i \in Q^{\text {no }}\right)}\right) \neq$ 
$\operatorname{th}^{d}\left(\sum_{i<r} M_{i}^{\mathrm{if}\left(i \in Q^{\mathrm{yes}}\right)}\right)$ for the probability distribution $\mu^{*}(I)$, under the assumption $Q^{\text {no }} \Delta Q^{\text {yes }} \subseteq J$.

3) Without loss of generality in 3.4, $r \leq 2 k+1$ (and even $r=k$ ).

Proof: Note that the number of possible $\left\langle\operatorname{th}^{d}\left(M_{i}^{\mathfrak{t}}\right): i, \mathfrak{t}\right\rangle$ is finite.

1) By (2). First draw $i \in J$ (equal probability) and then use $\operatorname{spr}(I, J \backslash\{i\}$ ). Alternatively let $J=\left\{i_{0}, \ldots, i_{\ell(*)-1}\right\}$, where $0 \leq i_{0}<\ldots<i_{\ell(*)-1}<r$, $I=\{0, \ldots, r-1\}$ so $|J|=\ell(*)$. Let $J_{\ell(*), \ell} \stackrel{\text { def }}{=} J \backslash\left\{i_{\ell}\right\}$ for $\ell<\ell(*)$. Clearly

$(*)_{1}$

$$
\begin{aligned}
& \operatorname{Prob}_{\mu(I, J)}\left(\operatorname{th}^{d}\left(\sum_{i<r} M_{i}^{\mathrm{if}\left(i \in Q^{\mathrm{no}}\right)}\right) \neq \operatorname{th}^{d}\left(\sum_{i<r} M_{i}^{\mathrm{if}\left(i \in Q^{\mathrm{yes}}\right)}\right) \mid\left(Q^{\mathrm{no}}, Q^{\mathrm{yes}}\right) \in \operatorname{spr}(I, J)\right) \\
& =\frac{1}{\ell(*)} \sum_{\ell<\ell(*)} 2^{-(r-1)}\left|\left\{Q \subseteq I \backslash\left\{i_{\ell}\right\}: \operatorname{th}^{d}\left(\sum_{i<r} M_{i}^{\mathrm{if}(i \in Q)}\right) \neq \operatorname{th}^{d}\left(\sum_{i<r} M_{i}^{\mathrm{if}\left(i \in Q \cup\left\{i_{\ell}\right\}\right)}\right)\right\}\right| .
\end{aligned}
$$

Hence if $\ell(*)=k+1$ then

$(*)_{2}$

$$
\begin{gathered}
\operatorname{Prob}_{\mu(I, J)}\left(\operatorname{th}^{d}\left(\sum_{i<r} M_{i}^{\mathrm{if}\left(i \in Q^{\mathrm{no}}\right)}\right) \neq \operatorname{th}^{d}\left(\sum_{i<r}\left(M_{i}^{\mathrm{if}\left(i \in Q^{\mathrm{yes}}\right)}\right) \mid\left(Q^{\mathrm{no}}, Q^{\mathrm{yes}}\right) \in \operatorname{spr}(I, J)\right)\right. \\
=\frac{1}{k+1} \sum_{\ell \leq k} \operatorname{Prob}_{\mu\left(I, J_{k+1, \ell}\right)}\left(\mathcal{E}_{\ell} \mid\left(Q^{\mathrm{no}}, Q^{\mathrm{yes}}\right) \in \operatorname{spr}\left(I, J_{\ell}\right)\right),
\end{gathered}
$$

where

$$
\mathcal{E}_{\ell}=\left\{\left(Q^{\mathrm{no}}, Q^{\mathrm{yes}}\right) \in \operatorname{spr}(I, J): \operatorname{th}^{d}\left(\sum_{i<r} M_{i}^{\mathrm{if}\left(i \in Q^{\mathrm{no}}\right)}\right)=\operatorname{th}^{d}\left(\sum_{i<r} M_{i}^{\mathrm{if}\left(i \in Q^{\mathrm{yes}}\right)}\right)\right\}
$$

Now compute.

2) Should be clear.

3) By addition theory i.e. 2.7(7) and by 2).

3.5 Definition: Let $c \in \mathbb{N}$ be the number of members in $\mathrm{TH}^{d}(0, \tau)$ (the set of formally possible $t h^{d}(\langle\rangle, M), M \in K$, see $\left.2.6(3)\right)$. Let $k_{0} \in \mathbb{N}$ be such that $k_{0} \rightarrow\left(3^{d+8}\right)_{c^{2}}^{2}$ (exists by Ramsey theorem).

\subsection{Observation:}

$(*)_{1} \quad \zeta_{k_{0}} \geq \frac{1}{\left(k_{0} 2^{k_{0}}\right)}$.

[Why? Let $r, M_{i}^{\mathfrak{t}}$, and $J,|J|=k_{0}$ be given. First draw $Q^{\text {yes,no }} \cap(r \backslash J)$ and 
assume they are equal.

Now (by $3.4 \mathrm{~A}(2)$ ) it is enough to prove that now the probability of the equality i.e. of

$$
\left.\operatorname{th}^{d}\left(\sum_{i<r} M_{i}^{\mathrm{if}\left(i \in Q^{\mathrm{no}}\right)}\right)=\operatorname{th}^{d}\left(\sum_{i<r} M_{i}^{\mathrm{if}\left(i \in Q^{\mathrm{yes}}\right)}\right)\right)
$$

is $\geq 1 /\left(k_{0} 2^{k_{0}}\right)$, assuming $Q^{\text {no }} \Delta Q^{\text {yes }}=\{j\} \subseteq J$. For $i<j$ from $J$ let $\left\langle\mathbf{t}_{i, j}^{\text {no }}, \mathbf{t}_{i, j}^{\text {yes }}\right\rangle$ be $\left\langle\operatorname{th}^{d}\left(M_{i}^{\mathrm{no}}+M_{i+1}^{\mathrm{no}}+\ldots+M_{j-1}^{\mathrm{no}}\right), \operatorname{th}^{d}\left(M_{i}^{\mathrm{yes}}+M_{i+1}^{\mathrm{no}}+M_{i+2}^{\mathrm{no}}+\ldots+M_{j-1}^{\text {no }}\right)\right\rangle$. So by the choice of $k_{0}$ we can find $J^{\prime} \subseteq J,\left|J^{\prime}\right|=3^{d+8}$ and $\left\langle\mathbf{t}_{0}, \mathbf{t}_{1}\right\rangle$ such that for $i<j$ in $J^{\prime}$, we have $\left\langle\mathbf{t}_{i, j}^{\text {no }}, \mathbf{t}_{i, j}^{\text {yes }}\right\rangle=\left\langle\mathbf{t}_{0}, \mathbf{t}_{1}\right\rangle$. Let $J^{\prime}=\left\{i_{\ell}: \ell<3^{d+8}\right\}$. For each $j \leq 3^{d+8}$ let $Q_{i}=\left\{i_{m}: m<j\right\}$. By addition theory for (first order theory) linear order, (that is $2.7(6))$ for $\ell \stackrel{\text { def }}{=}\left[3^{d+8} / 2\right], \operatorname{th}^{d}\left(\sum_{i} M_{i}^{\text {if }\left(i \in Q_{\ell}\right)}\right)=\operatorname{th}^{d}\left(\sum_{i} M_{i}^{\text {if }\left(i \in Q_{\ell+1}\right)}\right)$. So the probability for equality is at least the probability of $Q^{\text {no }}=Q_{\ell}, Q^{\text {yes }}=Q_{\ell+1}$ which is $\geq 1 /\left(k_{0} 2^{k_{0}}\right)$ ].

3.7 Observation: For every $\ell, k>0$ we have

$$
\xi_{k \ell} \leq \xi_{k}\left(\sum_{j \leq \ell-1}\left[\left(\begin{array}{c}
\ell-1 \\
j
\end{array}\right) \xi_{k}^{j}\left(1-\xi_{k}\right)^{\ell-1-j}\right] \xi_{j}\right)
$$

Proof: Let us be given $r, M_{i}^{\mathfrak{t}}$ for $i<r$ and $J$ as in $(*)$ so $|J|=k \ell$. Choose $\left\langle I_{j}: j<\ell\right\rangle$ a partition of $I \stackrel{\text { def }}{=} r=\{0, \ldots, r-1\}$ to intervals such that for $j<\ell$, $J_{j} \stackrel{\text { def }}{=} J \cap I_{j}$ has exactly $k$ members. Now first draw $Q_{j}^{\text {no }} \subseteq I_{j}$ for $j<\ell$ (with equal probabilities), second draw $s_{j} \in J_{j}$ for $j<\ell$ (with equal probabilities) and third draw $R^{\text {no }} \subseteq\{0, \ldots, \ell-1\}$ (with equal probabilities) and fourth draw $j(*)<\ell$ (with equal probabilities). Let $Q_{j}^{\mathrm{yes}}=Q_{j}^{\text {no }} \Delta\left\{s_{j}\right\}$, and $R^{\text {yes }}=R^{\text {no }} \Delta\{j(*)\}$, and $Q^{\text {no }}=\bigcup_{j<\ell} Q_{j}^{\mathrm{if}\left(\ell \in R^{\mathrm{no}}\right)}, Q^{\text {yes }}=\bigcup_{j<\ell} Q_{j}^{\mathrm{if}\left(\ell \in R^{\mathrm{yes}}\right)}$.

Easily $\left(Q_{j}^{\text {no }}, Q_{j}^{\text {yes }}\right)$ was chosen by the distribution $\mu^{*}\left(I_{j}, J_{j}\right)$ and $\left(R^{\text {no }}, R^{\text {yes }}\right)$ was chosen by the distribution $\mu^{*}(\{0, \ldots, \ell-1\})$ and $\left(Q^{\text {no }}, Q^{\text {yes }}\right)$ was chosen by the distribution $\mu^{*}(I, J)$. Hence it is enough to prove:

(*) $\operatorname{Prob}\left(\operatorname{th}^{d}\left(\sum_{i<r} M^{\mathrm{if}\left(i \in Q^{\mathrm{no}}\right)}\right) \neq \operatorname{th}^{d}\left(\sum_{i<r} M_{i}^{\mathrm{if}\left(i \in Q^{\mathrm{yes}}\right)}\right)\right)$

$\leq \xi_{k}\left(\sum_{j \leq \ell-1}\left[\left(\begin{array}{c}(\ell-1) \\ j\end{array}\right) \xi_{k}^{j}\left(1-\xi_{k}\right)^{\ell-1-j}\right] \xi_{j}\right)$.

For $j<\ell$ let $N_{i}^{\mathfrak{t}}=\sum_{i \in I_{j}} M^{\mathrm{if}\left(i \in Q_{j}^{\mathfrak{t}}\right)}$ and let $p_{i} \in[0,1]_{\mathbb{R}}$ be $\operatorname{Prob}\left(\operatorname{th}^{d}\left(N_{j}^{\mathrm{no}}\right) \neq\right.$ $\left.\operatorname{th}^{d}\left(N_{j}^{\text {yes }}\right)\right)$ and let $A=\left\{j: \operatorname{th}^{d}\left(N_{j}^{\text {no }}\right) \neq \operatorname{th}^{d}\left(N_{j}^{\text {yes }}\right)\right\}$, so the events " $j \in A$ " are independent and $p_{i}=\operatorname{Prob}(i \in A) \leq \xi_{k}$. Now if we make the first and second 
drawing only, we know $A,\left\langle N_{i}^{\mathfrak{t}}: i<\ell, \mathfrak{t} \in\{\right.$ no, yes $\left.\}\right\rangle$ and modulo this, by the definition of $\xi_{|A|}$ we know

$\operatorname{Prob}\left(\operatorname{th}^{d}\left(\sum_{i<r} M_{i}^{\mathrm{if}\left(i \in Q^{\mathrm{no}}\right)}\right) \neq \operatorname{th}^{d}\left(\sum_{i<r} M_{i}^{\mathrm{if}\left(i \in Q^{\mathrm{yes}}\right)}\right) \mid\right.$ after 1st and 2nd drawing $)=$

$\operatorname{Prob}\left(\operatorname{th}^{d}\left(\sum_{j<\ell} N_{j}^{\mathrm{if}\left(j \in R^{\mathrm{no}}\right)}\right) \neq \operatorname{th}^{d}\left(\sum_{j<\ell} N_{j}^{\mathrm{if}\left(j \in R^{\mathrm{yes}}\right)}\right) \mid\right.$ after 1st and 2nd drawing $) \leq \xi_{|A|} \cdot$

As the events " $j \in A$ " are independent we can conclude

$$
\begin{aligned}
& \operatorname{Prob}_{\mu^{*}(I, J)}\left(\operatorname{th}^{d}\left(\sum_{i<r} M_{i}^{\mathrm{if}\left(i \in Q^{\mathrm{no}}\right)}\right) \neq \operatorname{th}^{d}\left(\sum_{i<r} M_{i}^{\mathrm{if}\left(i \in Q^{\mathrm{yes}}\right)}\right) \mid\left(Q^{\mathrm{no}}, Q^{\mathrm{yes}}\right) \in \operatorname{spr}(I, J)\right) \\
& \leq \sum_{j \leq \ell} \operatorname{Prob}(|A|=j) \times \frac{j}{\ell} \times \xi_{j}=\sum_{j \leq \ell}\left(\sum_{u \subseteq \ell,|u|=j} \prod_{m \in u} p_{m} \prod_{m<\ell, m \notin u}\left(1-p_{m}\right)\right) \times \frac{j}{\ell} \xi_{j} .
\end{aligned}
$$

Now looking at this as a function in $p_{m} \in\left[0, \xi_{k}\right]_{\mathbb{R}}$ for $m<\ell$, for some $\left\langle p_{m}^{*}: m<\ell\right\rangle$ we get maximal values, and as the function is linear, $p_{m}^{*} \in\left\{0, \xi_{k}\right\}$, and as $0 \leq \xi_{j} \leq \xi_{j+1} \leq 1$, necessarily $p_{m}^{*}=\xi_{k}$ so

$$
\begin{gathered}
\operatorname{Prob}_{\mu^{*}(I, J)}\left(\operatorname{th}^{d}\left(\sum_{i<r} M_{i}^{\mathrm{if}\left(i \in Q^{\mathrm{no}}\right)}\right) \neq \operatorname{th}^{d}\left(\sum_{i<r} M_{i}^{\mathrm{if}\left(i \in Q^{\mathrm{yes}}\right)}\right) \mid\left(Q^{\mathrm{no}}, Q^{\mathrm{yes}}\right) \in \operatorname{spr}(I, J)\right) \\
\leq \sum_{j \leq \ell}\left(\sum_{u \subseteq \ell,|u|=1} \prod_{m \in u} p_{m}^{*} \prod_{m<\ell, m \notin u}\left(1-p_{m}^{*}\right)\right) \times \frac{j}{\ell} \times \xi_{j}= \\
=\sum_{j \leq \ell}\left(\begin{array}{c}
n \\
j
\end{array}\right)\left(\xi_{k}\right)^{j}\left(1-\xi_{k}\right)^{\ell-j}\left(\frac{j}{\ell}\right) \xi_{j}= \\
=\sum_{0<j \leq \ell}\left(\begin{array}{l}
\ell \\
j
\end{array}\right)\left(\xi_{k}\right)^{j}\left(1-\xi_{k}\right)^{\ell-j} \frac{j}{\ell} \xi_{j}= \\
=\sum_{j \leq \ell-1}\left[\left(\begin{array}{c}
\ell \\
j+1
\end{array}\right) \frac{(j+1)}{\ell}\left(\xi_{k}\right)^{j+1}\left(1-\xi_{k}\right)^{\ell-j-1}\right] \xi_{j}= \\
\xi_{k} \sum_{j \leq \ell-1}\left[\left(\begin{array}{c}
\ell-1 \\
j
\end{array}\right)\left(\xi_{k}\right)^{j}\left(1-\xi_{k}\right)^{(\ell-1)-j}\right] \xi_{j} .
\end{gathered}
$$

\subsection{Observation.}

1) If $\ell, k>0, j_{0} \leq \ell \xi_{k}$ then $\xi_{k \ell} \leq \xi_{k}\left(\frac{1+\xi_{j_{0}}}{2}\right)$.

2) If $\xi_{k} \leq 1-\frac{1}{m}, \ell>k / \xi_{k}$ then $\xi_{k \ell} \leq \xi_{k}\left(\frac{1+\xi_{k}}{2}\right)$. 
Proof: 1) As $\xi_{j} \leq \xi_{j+1}$ we have

$$
\begin{gathered}
\xi_{k \ell} \leq \xi_{k} \times\left(\sum_{j \leq \ell-1}\left[\left(\begin{array}{c}
\ell-1 \\
j
\end{array}\right)\left(\xi_{k}\right)^{j}\left(1-\xi_{k}\right)^{\ell-1-j}\right] \xi_{j}\right)= \\
=\xi_{k} \times\left(\sum_{j<j_{0}}\left[\left(\begin{array}{c}
\ell-1 \\
j
\end{array}\right)\left(\xi_{k}\right)^{j}\left(1-\xi_{k}\right)^{\ell-1-j}\right] \times 1+\right. \\
\left.\sum_{j \in\left[j_{0}, \ell-1\right)}\left[\frac{(\ell-1) !}{j !(\ell-j-1) !}\left(\xi_{k}\right)^{j}\left(1-\xi_{k}\right)^{\ell-1-j}\right] \times \xi_{j_{0}}\right)= \\
\leq \xi_{k}\left(\frac{1+\xi_{j_{0}}}{2}\right) .
\end{gathered}
$$

2) Follows.

3.8A Remark. Using "the binomial distribution approach normal distribution" and 3.6, clearly we get e.g.:

for every $\varepsilon>0$, for some $\ell_{\varepsilon}$, for every $\ell \geq \ell_{\varepsilon}$ we have

$$
\xi_{k \ell} \leq \xi_{k} \xi_{(1-\varepsilon) \ell}
$$

3.9 Proof of 3.3: 1) By the definition of $\zeta_{k}$ and Observations 3.5, 3.6 we get that $\lim _{k \rightarrow \infty} \zeta_{k}=1$ and we can finish easily.

2) Follows by (1) (and the addition theory see 2.7, particularly 2.7(7))

3) Similar proof and not used (e.g. imitate the proof of 3.6. First choose $j(*)$ then we have probability $\zeta_{\kappa}$ for equality there if the distribution is $\mu^{*}\left(I_{j(*)}, J_{j(*)}\right)$, but the induced distribution is very similar to it).

4) Follows very easily. For $\operatorname{spr}(k)$ with probability $1 / 2$ we are choosing by $\operatorname{npr}(k)$. $\square_{3.2}$

3.10 Proof of 1.4: Let a real $\varepsilon>0$ and a sentence $\theta \in \mathcal{L}_{\tau}^{\text {fo }}(\tau$-from 1.3) be given. We shall define $\varphi$ below (after $\left.(*)_{1}\right)$, and let $\psi=\psi_{\varphi}, \tau_{1}^{*}$ (a vocabulary) and $m \in \mathbb{N}$ and $\varphi_{i}(x)$ for $i<m$ be defined as in 2.16 (for the $\varphi$ here). Let $d$ be the quantifier depth of $\psi_{\varphi}$ (i.e. $d=d\left[\psi_{\varphi}\right]$ ). Let $k^{*} \in \mathbb{N}$ be large enough as in 3.3(4) (for the given $\varepsilon, m$ and $\tau_{1}^{*}$ ). Let $k$ be $\left(2 k^{*}+2\right)\left(3^{d[\theta]}+1\right)$ (we could have waived the $\left.3^{d[\theta]}+1\right)$. Now choose by induction on $r \leq k, m_{r} \in \mathbb{N}$ such that

(*) (a) $0=m_{0}$

(b) $m_{r}<m_{r+1}<\ldots$

(c) for any $n$, 


$$
\varepsilon / 3>\operatorname{Prob}_{\mu_{n}}\left(M_{n} \models \bigvee_{r}\left(\exists x \leq m_{r}+1\right)\left(\exists y \geq m_{r+1}-1\right)[x R y] \mid M_{n} \in K_{n}\right) .
$$

[Why this is possible? We choose $m_{r}$ by induction on $r$. The probability in question is, for each fixed $r$, bounded from above by $\sum_{i<m_{r}} \sum_{j>m_{r+1}} p_{j-i}$, the sum is the tail of an (absolutely) convergent infinite sum so by increasing $m_{r+1}$ we can make it $<\varepsilon / 2^{r+2}$, this suffices].

Next we try to draw the model $M_{n}$ in another way. Let $n>m_{k}$ be given; let $n^{*}=n+k^{*}+1$. Let $J=\left\{m_{\left(3^{d[\theta]+1}\right) i}: 0<i<2 k^{*}\right\}$, and $I=\left\{m_{i}: i<2 k^{*} 3^{d[\theta]+1}\right\}$. We first draw $\mathfrak{A}_{n}$, "a drunkard model $\mathfrak{A}_{n}$ for $n$ ". Drawing $\mathfrak{A}_{n}$ means: laziness case $=$ first case if $i<j<n^{*}, \bigvee_{r<k}\left[i \leq m_{r}+1 \& j \geq m_{r+1}-1\right]$ then $\{i, j\}$ is non edge (no drawing).

normal case $=$ second case: if $i<j<n^{*}$ are not in the first case but $\neg(\exists m \in$ $I)[i \leq m \leq j]$ then we flip a coin and get $e_{i, j} \in\{$ yes, no $\}$ with probability $p_{j-i}$ (for yes).

drunkard case=third case: $i<j<n^{*}$ and no previous case apply; we make two draws. In one we get $e_{i, j}^{1} \in\{$ yes, no $\}$ with probability $p_{j-i-1}$ (for yes) in the second we get $e_{i, j}^{2} \in\{$ yes, no $\}$ with probability $p_{j-i}$ for yes (we may stipulate $\left.p_{0}=0\right)$.

Now for every $Q \subseteq J$ we define $M_{Q}\left[\boldsymbol{\mathfrak { A }}_{n}\right]$ : it is a model $\left(A^{Q},<, P, R^{Q}\right)$ where $A^{Q}$ is $\left\{0, \ldots, n^{*}-1\right\} \backslash Q$ (so $\left\|M_{Q}\left[\mathfrak{\mathfrak { A }}_{n}\right]\right\|$ is $n^{*}-|Q|$ and usually $|Q|$ will be $k^{*}$ or $\left.k^{*}+1\right)$

$<$ is the usual order on $I^{Q}$

$P=\left\{m_{r}: r<k\right\}$

$R^{Q}$ is $^{\dagger}\left\{(i, j):\{i, j\}=\left\{i^{\prime}, j^{\prime}\right\}, i^{\prime}<j^{\prime}<n^{*}\right.$, and:

(a) $\left(i^{\prime}, j^{\prime}\right)$ fall into the second case above and $e_{i^{\prime}, j^{\prime}}=$ yes

or (b) $\left(i^{\prime}, j^{\prime}\right)$ fall into the third case say $i \leq m \leq j$ and $m \in I$

( $m$ is unique by " not first case") $m \in Q$, and $e_{i^{\prime}, j^{\prime}}^{1}=$ yes or (c) $\left(i^{\prime}, j^{\prime}\right)$ fall into the third case say $i \leq m \leq j$ and $m \in I$ ( $m$ is unique by " not first case") $m \notin Q$ and $e_{i^{\prime}, j^{\prime}}^{2}=$ yes $\}$.

We also define a model $N\left[\boldsymbol{A}_{n}\right]$ :

the universe: $\left\{0, \ldots, n^{*}-1\right\}$

relations: $<$ the usual order

$$
R=\left\{\langle i, j\rangle: e_{i, j}=\text { yes, } i<j\right\}
$$

$\dagger^{\dagger}$ We use $i, j$ so that without loss of generality $i^{\prime}<j^{\prime}$. 


$$
\begin{aligned}
& R^{1}=\left\{\langle i, j\rangle: e_{i, j}^{1}=\text { yes, } i<j\right\} \\
& R^{2}=\left\{\langle i, j\rangle: e_{i, j}^{2}=\text { yes, } i<j\right\} \\
& P=\left\{m_{r}: r \leq k\right\}(\text { on } \kappa \text { see above, before }(*))
\end{aligned}
$$

Observe

$(*)_{1}$ in $\left(N\left[\boldsymbol{\mathfrak { A }}_{n}\right], Q\right)$ we can define $M_{Q}\left[\boldsymbol{\mathfrak { A }}_{n}\right]$ by q.f. formulas.

So for some first order $\varphi$ depending on $\theta, \tau$ but not on $n$ (promised above in the beginning of the proof):

$(*)_{2} M_{Q}\left[\boldsymbol{\mathfrak { A }}_{n}\right] \models \theta \underline{\text { iff }}\left(N\left[\boldsymbol{\mathfrak { A }}_{n}\right], Q\right) \models \varphi$.

By 2.16 (where $I\left[\left(N\left[\boldsymbol{A}_{n}\right], Q\right)\right]$ is defined in 2.16 with $M$ there standing for $\left(N\left[\boldsymbol{\mathfrak { A }}_{n}\right], Q\right)$ here, so its set of elements is $\left.P\right)$ and the choice of $\psi_{\varphi}$ we have:

$(*)_{3}\left(N\left[\boldsymbol{\mathfrak { A }}_{n}\right], Q\right) \models \varphi$ iff $I\left[\left(N\left[\boldsymbol{\mathfrak { A }}_{n}\right], Q\right)\right] \models \psi_{\varphi}$.

Looking at the definition of $I\left[N\left[\boldsymbol{\mathfrak { A }}_{n}\right], Q\right]$ in 2.16 without loss of generality

$(*)_{4} I\left[\left(N\left[\boldsymbol{\mathfrak { A }}_{n}\right], Q\right)\right] \models \psi_{\varphi}$ iff $\left(I\left[N\left[\boldsymbol{\mathfrak { A }}_{n}\right]\right], Q\right) \models \psi_{\varphi}$.

Let $J=J^{d} \cup J^{u}, J^{d}$ an initial segment, $J^{u}$ an end segment, $\left|J^{u}\right|=k^{*},\left|J^{d}\right|=$ $k^{*}-1$. Now we define further drawing; let $\mu^{* *}\left[J, J^{u}\right]$ be the distribution from 3.2 above on $\operatorname{npr}\left(J^{u}\right)$, and choose $\left(Q_{0}^{u}, Q_{1}^{u}\right) \in \operatorname{npr}\left(J^{u}\right)$ randomly by $\mu^{* *}\left(J^{u}\right)$ then choose $Q_{1}^{d} \subseteq J^{d}$ such that $\left|Q_{1}^{d}\right|=k^{*}+1-\left|Q_{1}^{u}\right|$ with equal probabilities, and let $Q^{1}=Q_{1}^{d} \cup Q_{1}^{u}$, and let $Q_{0}^{d}=Q_{1}^{d}, Q^{0}=Q_{0}^{d} \cup Q_{0}^{u}$.

Now for $\ell \in\{0,1\}$ we make a further drawing: if $i<j$ is a pair from the first possibility (in the drawing of $\boldsymbol{A}_{n}$ ), we flip a coin for ${ }^{*} e_{i, j}^{\ell} \in\{$ yes, no $\}$ with probability $p_{\mid[i, j) \backslash Q^{\ell \mid}}$ for yes.

Let $M_{Q^{\ell}}^{\ell}$ be $\left(A^{Q^{\ell}},<, R^{Q^{\ell}} \cup\left\{(i, j),(j, i): i<j\right.\right.$ and: ${ }^{*} e_{i, j}^{\ell}=$ yes $\left.\}\right)$ (it depends on the choice of $\boldsymbol{\mathfrak { A }}_{n}$ and on the further drawing).

Now reflecting we see

$(*)_{5}$ for $\ell=0,1$, the distribution of $M_{Q^{\ell}}^{\ell}$ is the same as that of $\left(K_{n+1-\ell}, \mu_{n+1-\ell}\right)$ (from Def. 1.3).

Hence

$(*)_{6}$ for $\ell=0,1, \operatorname{Prob}\left(M_{Q^{\ell}}^{\ell} \models \theta\right)=\operatorname{Prob}\left(M_{n+1-\ell} \models \theta \mid M_{n+1-\ell} \in K_{n+1-\ell}\right)$.

By the choice of $m_{r}$ 's

$(*)_{7} \operatorname{Prob}\left(M_{Q^{\ell}}^{\ell}=M_{Q^{\ell}}\left[\boldsymbol{\mathfrak { A }}_{n}\right]\right)$ is $\geq 1-\varepsilon / 3$.

By 3.3 above (used above: the drawing of $\left(Q_{0}^{u}, Q_{1}^{u}\right)$ was randomly by $\left.\mu^{* *}\left(J^{u}\right)\right)$.

$(*)_{8}$ the absolute value of the differences between the following is $\leq \varepsilon / 3$ :

$\left.\operatorname{Prob}\left(\left[N\left[\boldsymbol{\mathfrak { A }}_{n}\right]\right], Q^{0}\right) \models \psi_{\varphi}\right)$

$\left.\operatorname{Prob}\left(\left[N\left[\boldsymbol{\mathfrak { A }}_{n}\right]\right], Q^{1}\right) \models \psi_{\varphi}\right)$.

So for $\ell=0,1$ :

(a) $\operatorname{Prob}\left(M_{n+\ell} \models \theta \mid M_{n+\ell} \in K_{n+\ell}\right)=\operatorname{Prob}\left(M_{Q^{\ell}}^{\ell} \models \theta\right)$

[Why? by $\left.(*)_{6} \cdot\right]$ 
(b) $\operatorname{Prob}\left(M_{Q^{\ell}}^{\ell}=M_{Q^{\ell}}\left[\boldsymbol{\mathfrak { A }}_{n}\right]\right) \geq 1-\varepsilon / 3$

[Why? by $\left.(*)_{7} \cdot\right]$

(c) $M_{Q^{\ell}}\left[\boldsymbol{\mathfrak { A }}_{n}\right] \models \theta$ iff $\left(I\left[N\left[\boldsymbol{\mathfrak { A }}_{n}\right]\right], Q^{\ell}\right) \models \psi_{\varphi}$

[Why? by $(*)_{1}+(*)_{2}+(*)_{3}+(*)_{4}$.]

By $(\mathrm{a})+(\mathrm{b})+(\mathrm{c})$ it suffices to prove that the probabilities of

$$
\text { "(I[N[} \left.\left.\left.\boldsymbol{\mathfrak { A }}_{n}\right]\right], Q^{\ell}\right) \models \psi_{\varphi} "
$$

for $\ell=0,1$ has difference $<\varepsilon / 3$ but this holds by $(*)_{8}$.

\section{$\S 4$ Generalized sums and Distortions:}

We try here to explain the results on $\S 2$ as distorted generalized sums (and the connection with generalized sums) and later the connection to models with distance. First we present for background the definition and theorem of the generalized sum.

4.1 Definition: Let $\tau_{0}, \tau_{1}, \tau_{2}$ be vocabularies of models. For a $\tau_{0}$-model $I$ (serving as an index model $), \tau_{1}$-models, pairwise disjoint for simplicity $M_{t}(t \in I)$, and function $F$ (explained below) we say that a $\tau_{2}$-model $M$ is the $F$-sum of $\left\langle M_{t}\right.$ : $t \in I\rangle)$ in symbols $M=\oplus_{F}\left\{M_{t}: t \in I\right\} \underline{\text { if: }}$

(a) the universe $|M|$ of $M$ is $\bigcup_{t \in I}\left|M_{t}\right|$ (if the $M_{t}$ 's are not pairwise disjoint: $\left.\left\{(t, a): t \in I, a \in M_{t}\right\}\right)$ and we define $h_{\alpha}: M \rightarrow I, h(a)=t$ if $a \in M_{t}$ (if not disjoint $h(\langle t, a\rangle)=t)$,

(b) if $t_{1}, \ldots, t_{k} \in I$ are pairwise distinct, $\bar{a}=\bar{a}_{1} \widehat{\ldots} \widehat{a}_{k}$ and $\bar{a}_{\ell} \in M_{t_{\ell}}$ (finite sequences) then

$$
\operatorname{tp}_{\mathrm{qf}}(\bar{a}, \emptyset, M)=F\left(\operatorname{tp}_{\mathrm{qf}}\left(\left\langle t_{1}, \ldots, t_{k}\right\rangle, \emptyset, I\right), \operatorname{tp}_{\mathrm{qf}}\left(\bar{a}_{1}, \emptyset, M_{t_{1}}\right), \ldots, \operatorname{tp}_{\mathrm{qf}}\left(\bar{a}_{k}, \emptyset, M_{t_{k}}\right)\right) .
$$

Another way to say it is:

(b) ${ }^{\prime}$ if $a_{0}, \ldots, a_{k-1} \in M$ then $\operatorname{tp}_{\mathrm{qf}}\left(\left\langle a_{0}, \ldots, a_{k-1}\right\rangle, \emptyset, M\right)=$

$F\left(\operatorname{tp}_{\mathrm{qf}}\left(\left\langle h\left(a_{\ell}\right): \ell<k\right\rangle\right), \ldots, \operatorname{tp}_{\mathrm{qf}}\left(\left\langle a_{\ell}: \ell<k, t=h\left(a_{\ell}\right)\right\rangle, \emptyset, M_{t}\right), \ldots\right)_{t \in\left\{h\left(a_{\ell}\right): \ell<k\right\}}$ 
4.2 Remark: 1) So the form of $F$ is implicitly defined, it suffices to look at sequences $\bar{a}_{0} \widehat{ } \ldots \bar{a}_{k}$ (in clause $(\mathrm{b})$ ) or $\left\langle a_{0}, \ldots, a_{k}\right\rangle$ of length the arity of $\tau_{2}$ (if it is finite) i.e. maximum numbers of places for $P$ predicates $P \in \tau_{2}$.

2) We can consider a generalization where the universe of $M$ and equality are defined like any other relation.

4.3 The generalized Sum Theorem: In the notation above if $\boldsymbol{\Delta}_{n}$ is the set of formulas of quantifier depth $n$ then we can compute from $F$ the following function: like $F$ in (b) (or (b)') replacing tp $\operatorname{tp}_{\mathrm{qf}}$ by $\boldsymbol{\Delta}_{n}$.

4.4 Discussion: So looking at a sequence $\bar{a}$ from $M$, to find its [quantifier free] types we need to know two things:

$(\alpha)$ the [quantifier free] type of its restriction to each $h^{-1}(\{t\})=\{b \in M: h(b)=$ $t\}$

$(\beta)$ the [quantifier free] type of the sequence of parts, $\left\langle h\left(b_{\ell}\right): \ell<k\right\rangle$ in $I$.

4.5 Definition: Now $M$ is a $d$-distorted $F$-sum of $\left\{M_{t}: t \in I\right\}$ if

(a) $d$ is a distance function on $I$.

(b) $|M|$ is the disjoint union of $A_{t}(t \in I)$ and for each $t$ we have a model $M_{t}$ with universe $\cup\left\{A_{s}: s \in I, d(s, t) \leq 1\right\}$ and

$(\mathrm{c})^{+}$if $b_{0}, \ldots, b_{k-1} \in M$, then

$$
\begin{aligned}
& \operatorname{tp}_{\mathrm{qf}}\left(\left\langle b_{0}, \ldots, b_{k-1}\right\rangle, \emptyset, M\right)= \\
& F\left(\operatorname { t p } _ { \mathrm { qf } } \left(\left\langle h\left(b_{0}\right) \ldots, h\left(b_{k-1}\right), \emptyset, I\right), \ldots,\right.\right. \\
& \left.\quad \operatorname{tp}_{\mathrm{qf}}\left(\left\langle b_{\ell}: \ell<k, d\left(h\left(b_{\ell}\right), t\right) \leq 1\right), \emptyset, M_{t}\right), \ldots\right)_{t \in\left\{h\left(b_{m}\right): m<k\right\}}
\end{aligned}
$$

4.6 Remark: Note: by $\left\langle b_{\ell}: \ell<k, \operatorname{Pr}(\ell)\right\rangle$ we mean the function $g$ with domain $\{\ell<k: \operatorname{Pr}(\ell)\}$, satisfying $\left.g(\ell)=b_{\ell}\right)$.

4.7 Discussion: Our main Lemma 2.14, generalizes the generalized sum theorem, to distorted sum but naturally the distortion "expands" with the quantifier depth.

4.8 The Distorted Sum Generalized Lemma: In the notation above, for $m$ let $M_{t}^{m}$ be the model with

universe: $\cup\left\{A_{s}: d(s, t) \leq m\right\} \cup\{s \in I: d(s, t) \leq m\}$ 
relations: those of the $M_{s}$ 's i.e. for $R \in \tau_{1}$, a $k$-place predicate we let

$$
\begin{gathered}
Q_{R}^{t, m}=\left\{\left\langle s, a_{1}, \ldots, a_{k}\right\rangle: s \in I, d(s, t) \leq m+1,\left\langle a_{1}, \ldots, a_{k}\right\rangle \in R^{M_{s}}\right\} \\
\left(\text { so }\left\{a_{1}, \ldots, a_{k}\right\} \subseteq \cup\left\{A_{s}: d(s, t) \leq 1\right\}\right) \\
Q_{d}^{t, \ell, m}=\left\{\left(s_{1}, s_{2}\right): d\left(s_{1}, t\right) \leq \ell \text { and } d\left(s_{2}, t\right) \leq \ell, d\left(s_{1}, s_{2}\right) \leq m\right\}, \\
Q_{h}=\left\{(s, a): a \in A_{s}, s \in I, d(s, t) \leq m\right\} .
\end{gathered}
$$

We define $I^{[m, n]}$ as the expansion of $I$ by:

for $i \leq m$ and $\varphi \in \Delta_{n}\left(\tau_{0}\right)$

$$
Q_{\varphi}^{\ell}=\left\{t \in I: M_{t}^{\ell} \models \varphi\right\}
$$

Now there are functions $F_{n}$ and a number $m(n)=3^{n}$ computable from $F$ (and n) such that:

$\otimes$ for $b_{0}, \ldots, b_{k-1} \in M$

$$
\begin{array}{r}
\operatorname{tp}_{\boldsymbol{\Delta}_{n}}\left(\left\langle b_{0}, \ldots, b_{k-1}\right\rangle, \emptyset, M\right)=F_{n}\left(\operatorname{tp}_{\boldsymbol{\Delta}_{n}}\left(\left\langle h\left(b_{0}\right), \ldots, h\left(b_{k-1}\right)\right\rangle, \emptyset, I^{[m(n), n]}\right), \ldots,\right. \\
\left.\operatorname{tp}_{\boldsymbol{\Delta}_{n}}\left(\left\langle b_{\ell}: \ell<k, d\left(h\left(b_{\ell}\right), t\right) \leq m(n)\right\rangle, \emptyset, M_{t}^{m(n)}\right), \ldots\right)_{t \in\left\{h\left(b_{m}\right): m<k\right\}} .
\end{array}
$$

4.9 Discussion: 1) Now if $d$ is trivial:

$$
d(x, g)= \begin{cases}0 & x=y \\ \infty & x \neq y\end{cases}
$$

then $M_{t}^{n}=M_{t}$, and 2.8 (the disorted generalized sum Lemma) becomes degenerated to 4.3 (the generalized sum Lemma), more exactly a variant.

2) Note that 4.8 improve on the result of $\S 2$ in $m(n)$ not depending on $k$. We can have this improvement in $\S 2$ and in $\S 5$.

3) To prove 4.8 given $b_{0}, \ldots, b_{k-1}$ and looking for $y$ with

$$
\mathbf{t}=\operatorname{tp}_{\boldsymbol{\Delta}_{n+1}}\left(\left\langle b_{0}, \ldots, b_{k-1}, y\right\rangle, \emptyset, M\right)
$$

we fix $w=\left\{\ell<k: d\left(h\left(b_{\ell}\right), h(y)\right) \leq m(n)\right\}$.

E.g. if $w \neq \emptyset$ and $\ell(*)=\min (w)$, then the relevant properties of $y$ are expressed in the balls

$$
\left\{z \in M: d\left(h(z), h\left(b_{\ell}\right)\right) \leq m(n)\right\}
$$


for $\ell \in w$ and

$$
\{z \in M: d(h(z), h(y)) \leq m(n)\},
$$

all included in the ball

$$
\left\{z \in M: d\left(h(z), h\left(b_{\ell(*)}\right)\right) \leq 3 m(n)\right\}
$$

The case $w=\emptyset$ is simpler.

\section{$\S 5$ Models with Distance}

5.1 Discussion: We try here to explain the results of $\S 2$ as concerning a model with a distance function "weakly suitable" for the model and the connection to models with a distance function for the whole vocabulary which is suitable for the model. This is another variant of the distorted sums.

5.2 Context: Let $\tau$ be a fixed vocabulary.

1) Let $K$ be the class of $\mathfrak{A}=(M, d), M$ a $\tau$-model, $d$ a distance on $M$ (i.e. a two place symmetric function from $|M|$ to $\omega \cup\{\infty\}, d(x, x)=0$, satisfying the triangular inequality) and for simplicity $d(x, y)=0 \Leftrightarrow x=y$.

2) $K^{\text {sut }} \subseteq K$ is the class of $(M, d) \in K$ such that $d$ (which is a distance on $M$ ) is suitable for the model, i.e.

$\otimes_{1}\left\langle a_{0}, \ldots, a_{k-1}\right\rangle \in R^{M}, R \in \tau \Rightarrow \bigwedge_{\ell<m<k} d\left(a_{\ell}, a_{m}\right) \leq 1$.

3) $K^{\text {sim }} \subseteq K$ is the class of $(M, d) \in K$ which are simple, i.e

$\otimes_{2} d(x, y)=\operatorname{Min}\left\{n:\right.$ there are $z_{0}, \ldots, z_{n}$ such that $x=z_{0}, z_{n}=y$ and

$$
\left.\bigwedge_{\ell<n} \bigvee_{R \in \tau}\left(\exists \bar{a} \in R^{M}\right)\left[\left\{z_{\ell}, z_{\ell+1}\right\} \subseteq \operatorname{Rang} \bar{a}\right]\right\}
$$

4) $K_{F}^{\mathrm{ws}}$ is the class of $(M, d) \in K$ which are $F$-weakly suitable which means:

$\otimes_{3}$ for every $m_{i}$ if $\bar{a}^{i}=\left\langle a_{0}^{i}, \ldots, a_{n_{i}-1}^{i}\right)$ for $i<k, a_{\ell}^{i} \in M, d\left(a_{0}^{i}, a_{\ell}^{i}\right) \leq m_{i}$ and $i<j<k \Rightarrow d\left(a_{0}^{i}, a_{0}^{j}\right)>m_{i}+m_{j}+1$ then the quantifier free type of $\bar{a}^{0 \wedge} \bar{a}^{1 \curlywedge} \ldots \bar{a}^{k-1}$ is computed by $F$ for the quantifier free types of $\bar{a}^{0}, \bar{a}^{1}, \ldots, \bar{a}^{k-1}$ and of $\left\langle a_{0}^{0}, a_{0}^{1}, \ldots, a_{0}^{k-1}\right\rangle$

Note: we can strengthen the demands e.g. (for $f$ as in 2.5)

(*) $d\left(a_{0}^{i}, a_{\ell}^{i}\right) \leq r_{i}, d\left(a_{0}^{i}, a_{0}^{j}\right)>f_{0}\left(r_{i}\right)+f_{0}\left(r_{j}\right)+1$ or at least $\neg(\exists x, y)\left[d\left(x, a_{i}\right) \leq\right.$ $\left.f_{0}\left(r_{i}\right) \wedge d\left(y, a_{j}\right) \leq f_{0}\left(r_{j}\right) \wedge d(x, y) \leq 1\right]$.

5) $K^{\text {as }}$ is the family of $\mathfrak{B}=(M, d) \in K$ which are almost simple: " $d(x, y) \leq 1$ " is defined by quantifier free formula and $d(x, y)=\operatorname{Min}\left\{n\right.$ : we can find $z_{0}, \ldots, z_{n}, x=$ $\left.z_{0}, z_{n}=y, d\left(z_{\ell}, z_{\ell+1}\right) \leq 1\right\}$. 
5.3 Discussion: For $(M, d) \in K_{F}^{\mathrm{ws}}$ we want to "separate" the quantification to bounded ones and to distant ones. We can either note that it fits the context of $\S 2$ or repeat it.

5.4 Definition: 1) For $\mathfrak{B}=(M, d) \in K, x \in M, m<\omega$ let $N_{m}^{+}(x)=\{y \in M$ : $d(y, x) \leq m\}$.

2) We define " $\bar{a}$ is a $(\mathfrak{B}, r)$-component" and $\operatorname{bth}_{r}^{n}(\bar{a}, \mathfrak{B})$ as in Definition 2.8(1), and $\mathrm{BTH}_{r}^{n}(m, \tau)$ as in 2.8(2).

3) We define $\boldsymbol{B}_{n, m}^{2}$ as expanding $\mathfrak{B}$ by the relation $R_{\mathbf{t}}=\{\bar{a}: \bar{a}$ is a $(\mathfrak{B}, r)$ component, $\left.\mathbf{t}=\operatorname{bth}_{r}^{n}(\bar{a}, \mathfrak{B})\right\}$ for $\mathbf{t} \in \mathrm{BTH}_{r}^{n}(m, \tau)$.

4) We define " $\bar{a}$ is $(\mathfrak{B}, \bar{r})$-sparse" and $\operatorname{uth}_{\bar{r}}^{n}(\bar{a}, \mathfrak{B}) ", \operatorname{UTH}_{\bar{r}}^{n}(m, \tau)$ as in 2.12 .

5.5 Theorem: the parallel of 2.14 .

\section{References}

[B] E.W. Beth, Observations metamathematiques sur les structures simplement ordonnes, Collection de Logique Mathematique, Serie A, Fasc 5, Paris-Louvain 1954, 29-35.

[CK] Chang and Keisler, Model theory, North Holland Publishing Co, 1973, 1977.

[Gf] H. Gaifman, On local and non local properties, Proc. Herbrand Symp. Logic Colloquium, 81, ed. J. Stern; Studies in Logic Found. of Math vol 107 North Holland (1982) 105-135.

[Gu] Y. Gurevich, Monadic second order theories, Chap XIII, Model theoretic logics, ed. Barwise and Feferman Springer Verlag (1985); 479-506.

[Lu] T. Luczak, notes.

[LcSh 435] T. Luczak and S. Shelah, Convergence in homogeneous random graphs, Random Structures \& Algorithms, 1995, in press.

[ShSp 304] S. Shelah J. Spencer, On zero-one laws for random graph, Journal of A.M.S vol. 1 (1988) 97-115.

[Mo] A. Mostowski, On direct products of theories, Journal of Symbolic Logic, vol 17 (1952) 1-31.

[Mr] L. Marcus, Minimal models of theories of one function symbol, Israel J. of Math vol 18 (1974) 117-131.

[Sh 42] S. Shelah, The monadic theory of order, Annals of Mathematics, vol 102 (1975), 379-419.

[Sh F-120] S. Shelah, More on 0-1 laws, unpublished notes. 
[Sh 467] S. Shelah, More on random finite models, in preparation.

[Sh 548] S. Shelah, Very weak zero one law for random graphs with order and random binary functions, Random Structures $\&$ Algorithms, submitted.

[Sp] J. Spencer, Survey/expository paper: zero one laws with variable probabilities, Journal of Symbolic Logic vol 58 (1993) 1-14.

[Sh 550] S. Shelah, 0-1 laws, in preparation.

[Sh 551] S. Shelah, In the random graph $G(n, p), p=n^{-a}$ : if $\psi$ has probability $0\left(n^{-\varepsilon}\right)$ for every $\varepsilon>0$ then it has probability $0\left(e^{-n^{\varepsilon}}\right)$ for some $\varepsilon>0$, in preparation.

[F] R. Fagin, Probabilities in finite models, Journal of Symbolic Logic vol 45 (1976) 129-141

[FV] S. Feferman and R. L. Vaught, The first-order properties of algebraic systems, Fundamenta Mathematicae vol 47 (1959) 57-103

[GKLT] Y.V. Glebskii, D.I. Kogan, M.I. Liagonkii and V.A. Talanov, Range and degree of reliability of formulas in restricted predicate calculus Kibernetica vol 5 (1969) 17-27; translation of Cybernetics vol 5 pp 142-154. 